\title{
Catálogo general de las obras musicales de León Schidlowsky
}

\author{
por \\ Dr. David Schidlowsky \\ Berlín, Alemania \\ david@schidlowsky.com
}

\section{A. LISTA DE OBRAS SEGÚN CLASIFICACIÓN POR GÉNEROS MUSICALES, AÑO DE ESCRITURA Y NÚMERO EN EL CATÁLOGO CRONOLÓGICO}

\author{
Opera \\ Die Menschen \\ Der Dibbuk \\ Before Breakfast

\section{Orquesta} \\ Requiem (auf den Tod eines Knaben) \\ Oda a la tierra \\ Triptico \\ La noche de cristal \\ Invocación \\ Amereida \\ Llaqui \\ Memento \\ Ecce Homo \\ Nueva York \\ Deutsches Tagebuch \\ Monumento a Bolivar. \\ Kaddish \\ Epitafio para Hermann Scherchen \\ Akiba Ben Yosef \\ In Eius Memoriam \\ Monumentum \\ Introitus \\ Homenaje a Neruda \\ Hommage a Schoenberg \\ Trilogy \\ Prelude to a Drama \\ Lux in tenebris
}

$\begin{array}{lc}\text { Año } & \text { Número del catálogo } \\ 1969 & \text { O-94 } \\ 1993 & \text { O-259 } \\ 1998 & \text { O-282 }\end{array}$

$\begin{array}{lc}\text { Año } & \text { Númerodel catálogo } \\ 1954 & \text { O-13 } \\ 1958 & \text { O-40 } \\ 1959 & \text { O-48 } \\ 1961 & \text { O-50 } \\ 1964 & \text { O-56 } \\ 1965-1969 & \text { O-57 } \\ 1965 & \text { O-58 } \\ 1967 & \text { O-59 } \\ 1969 & \text { O-60 } \\ 1965 & \text { O-61 } \\ 1965 & \text { O-62 } \\ 1966 & \text { O-66 } \\ 1966-7 & \text { O-69 } \\ 1967 & \text { O-73 } \\ 1972 & \text { O-115 } \\ 1973 & \text { O-131 } \\ 1973 & \text { O-132 } \\ 1974 & \text { O-145 } \\ 1975 & \text { O-158 } \\ 1975 & \text { O-161 } \\ 1976 & \text { O-174 } \\ 1976 & \text { O-175 } \\ 1977 & \text { O-176 }\end{array}$




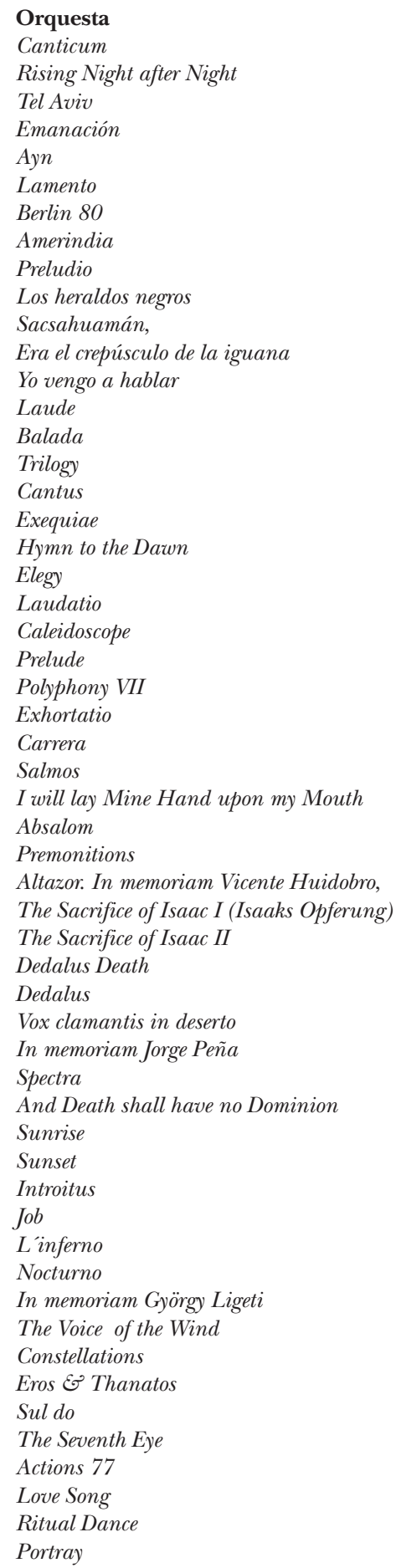

\begin{tabular}{|c|c|}
\hline Año & Número del catálogo \\
\hline 1978 & O-177 \\
\hline 1977 & O-179 \\
\hline $1978-83$ & O-183 \\
\hline 1979 & O-184 \\
\hline 1979 & O-186 \\
\hline 1980 & O-204 \\
\hline 1980 & O-206 \\
\hline $1982-85$ & O-217 \\
\hline 1982 & O-218 \\
\hline 1983 & O-219 \\
\hline 1983 & O-220 \\
\hline 1985 & O-221 \\
\hline 1983 & O-222 \\
\hline 1984 & O-227 \\
\hline 1985 & $\mathrm{O}-230$ \\
\hline 1986 & O-232 \\
\hline 1986 & O-233 \\
\hline 1986 & O-234 \\
\hline 1986-7 & O-235 \\
\hline 1988 & O-238 \\
\hline 1988 & O-239 \\
\hline 1989 & O-243 \\
\hline 1990 & O-245 \\
\hline 1991 & O-254 \\
\hline 1991 & O-255 \\
\hline 1991 & O-256 \\
\hline 1992 & O-257 \\
\hline 1994 & O-261 \\
\hline 1995 & O-265 \\
\hline 1997 & O-281 \\
\hline 1999 & O-286 \\
\hline 1999 & O-288 \\
\hline 1999 & O-290 \\
\hline 2000 & O-295 \\
\hline 2000 & O-296 \\
\hline 2000 & O-297 \\
\hline 2001 & O-301 \\
\hline 2002 & O-302 \\
\hline 2002 & O-309 \\
\hline 2004 & O-316 \\
\hline 2004 & O-317 \\
\hline 2004 & O-319 \\
\hline 2004 & O-326 \\
\hline 2005 & O-328 \\
\hline 2006 & O-334 \\
\hline 2006 & O-335 \\
\hline 2006 & O-337 \\
\hline 2006 & O-338 \\
\hline 2007 & O-339 \\
\hline 2007 & O-342 \\
\hline 2008 & O-343 \\
\hline 2008 & O-347 \\
\hline 2008 & O-350 \\
\hline 2009 & O-351 \\
\hline 2009 & O-353 \\
\hline
\end{tabular}




Orquesta
Lautaro
Mahleriana
Capricho
Obelisk
Desierto
Hopeless Day
Musical Landscapes
The Day of Rage
Memorial of Yesterday's Dreams
Presage
Epigram
The Valley of Dry Bones
Journey to the End of the Night
In memoriam Fernando Rosas
Valparaíso
Luces y sombras,
The World of the See
Memoria
Obelisk II
Partita- esquema para orquesta

Música de cámara

Elegía

Trio

Música 1956

Cuarteto mixto $N 1$

Cuarteto mixto $N 2$

Cuatro epigramas

Musica para ballet infantil

Concierto para seis instrumentos

Cuatro miniaturas (para cuarteto de vientos)

In memoriam

In memoriam

Soliloquios,

Eróstrato,

Cuarteto de cuerdas

Visiones

Quinteto de vientos,

Seis Hexáforos para Juan Manuel

Recital poético-musical

Sexteto

Babi Yar

Serenata

Invention

Images

Aura

Eclipse

Piano Quartet

Cuarteto de cuerdas

Clamavi

Shadows

In memoriam Luigi Nono

Septimino
Año

2009

2011

2011

2012

2012

2013

2013

2013

2013

2013

2013

2014

2014

2014

2014

2015

2015

2015

2015

2015

Año

1952

1955

1956

1956

1956

1956

1956

1957

1957

1957

1958

1961

1962

1967

1967

1968

1968

1968

1970

1970

1970

1976

1976

1978

1983

1988

1989

1990

1990

1990

1991
Número del catálogo

O-354

O-355

O-357

O-361

O-367

O-370

O-371

O-372

O-373

O-374

O-375

O-376

O-377

O-378

O-379

O-380

O-381

O-382

O-384

O-385

Número del catálogo

O-7

O-16

O-23

O-28

O-29

O-30

O-31

O-33

O-34

O-35

O-42

O-51

O-53

O-74

O-75

O-76

O-77

O-82

O-95

O-96

O-97

O-163

O-178

O-182

O-223

O-240

O-241

O-246

O-247

O-248

O-252 
Música de cámara

Sealed Room

In memoriam

Threnos (trio)

The Darkest Night

De profundis

Seven Nocturnes

Mahnmal-Memorial

El muro

Meditaciones

Evocación

Tenebrae

Duo

Transparencias

Adagio

Trio "The Swan"

The Secret Garden

Coming Back to Life, Tango

Septet

Quartet

Quintet

Elegie

Sextett

Resonances

Toccata

The March of the Ants

Twilight

Intermezzo

Sexteto

Chamber Concert (for 14 players)

Vocal

Cinco poemas para contralto y piano

Zwei Lieder vom Tode

Drei Liebeslieder

Tres canciones bíblicas

Dos Salmos

Salmo

5 composiciones

Sechs japanische Lieder

Tres canciones (también titulada Tres poemas)

Tres canciones

Altazor VII Canto

Cantata negra

Cinco canciones de amor

Caupolicán

Epitafio a Vicente Huidobro

Zwei Lieder

Amatorias

De profundis,

Cuatro episodios

Tres versos del capitán

Jeremias

Estudiante baleado
Año

1993

1996

1996

1997

1997

1998

1999

1999

2000

2000

2000

2001

2002

2002

2002

2003

2003

2004

2004

2004

2004

2004

2005

2005

2007

2011

2012

2012

2015

Año

1952

1952

1953

1954

1954

1954

1954

1954

1955

1955

1956

1957

1957

1958

1959

1962

1962-63

1963

1966

1966

1966

1967
Número del catálogo

O-260

O-266

O-267

O-268

O-269

O-283

O-287

O-289

O-291

O-293

O-294

O-298

O-303

O-304

O-308

O-310

O-312

O-318

O-321

O-324

O-325

O-327

O-330

O-331

O-341

O-358

O-359

O-360

O-383

Número del catálogo

O-4

O-8

O-9

O-10

O-11

O-12

O-14

O-15

O-17

O-18

O-27

O-37

O-38

O-41

O-47

O-52

O-54

O-55

O-64

O-65

O-67

O-71 


\section{Vocal}

Eclosión

Espergesia

Autorretrato del poeta

Grabstein für Else Lasker-Schüler

Grabstein für Else Lasker-Schüler

Lyrik

Dialog mit Martin Buber

In memoriam (Azkarat neshamot),

Eleven Tombstones

5 Impromtus pour la Reine Blanche

Shoa

Cuatro canciones

Adieu

Ode

Missa in Nomine Bach

Silvestre Revueltas

Lamento

Si muero

Bakasha

The Song of Sorrow

Four Songs (cuatro canciones)

Two Songs

Two Songs

Horror Vacui

Three Songs

Soledad

$Y$ alzase el hombre

\section{Coral}

Canciones de amor

Three Hebrew Pieces

Lamentación

Imprecaciones

Requiem para coro a cappella,

Eli Eli lama azavtani (Mein Gott, mein Gott, warum hast

Du mich verlassen) (Oh Dios, Oh Dios, porque me has abandonado),

Bereshit (=en el principio)

Nacht

Der heilige Sand

Bereshit

Missa Dona Nobis Pacem

An den Knaben Elis

Todesfuge

Laudate

Three Psalms and a Prayer

Libera me Domini

Three Pieces

Two Psalms

Solo

Exodus

Gedanken-Thoughts (Pensamientos)
Año

1967

1968

1968

1969

1969

1969

1969

1971

1972

1974

1975

1980

1982

1982

1984

1994

1998

1998

2001

2003

2003

2005

2006

2007

2008

2009

2012

Año

1957

1965-6

1966

1967

1968

1969

1969

1979

1980

1985

1987

1989

1991

1995

2004

2004

2005

2008

2011

2012

2015
Número del catálogo

O-72

O-79

O-81

O-83

O-84

O-85

O-86

O-101

O-121

O-143

O-159

O-205

O-213

O-215

O-226

O-263

O-284

O-285

O-299

O-311

O-313

O-333

O-336

O-340

O-344

O-352

O-363

Número del catálogo

O-36

O-63

O-68

O-70

O-78

O-87

O-88

O-185

O-207

O-231

O-237

O-242

O-253

O-264

O-320

O-322

O-332

O-349

O-356

O-386

O-366 


\section{Piano}

Música para mi mamá

Apología hebrea

Olga

Tres trozos

Seis miniaturas

Ocho estructuras

Cinco piezas

Actus

Trilogy (2 pianos)

Toccata

Violín

Three Dialogues

Prayer

Shakesperiana (dos voces y dos violines)

Izkor

Cello

Cadencia,

Partita

Guitarra y Mandolina

Interludio (guitarra)

Serenata (mandolina)

Intermezo (cuarteto mandolinas)

Monolog (guitarra y narrador)

Diagram, (mandolina y guitarra)

Prelude (mandolina)

Arpas

Five Pieces for Harp

Duetto (dos arpas)

Flauta

Isla negra

Arabesque

Farewell

Dueto (dos flautas)

\section{Clarinete}

Morgengebet,

Partita

Oboe

Partita

Órgano

Christus im Holocaust

Electroacústica

Nacimiento

Citizen 1230316 (Radiophonic poem)
Año

1943

$1947-48$

1948

1952

1952

1955

1956

1972

1990

1991

Año

2000

2002

2012

2012

Año

1961

2005

Año

1968

2002

2004

2008

2008

2008

Año

1989

2004

Año

1959

1991

2012

2012

Año

1993

2004

Año

2002

Año

2001

Año

1956

1975
Número del catálogo

O-1

O-2

O-3

O-5

O-6

O-19

O-32

O-116

O-249

O-250

Número del catálogo

O-292

O-306

O-362

O-365

Número del catálogo

O-49

O-329

Número del catálogo

O-80

O-307

O-314

O-345

O-346

O-348

Número del catálogo

O-244

O-315

Número del catálogo

O-43

O-251

O-364

O-368

Número del catálogo O-258

O-323

Número del catálogo

$$
\text { O-305 }
$$

Número del catálogo

O-300

Número del catálogo

O-24

O-160 


\begin{tabular}{|c|c|c|}
\hline Gráfica-musical / Multimedia ${ }^{1}$ & Año & Número del catálogo \\
\hline Konstellation I & 1969 & O-89 \\
\hline Sprüche, (3 partes) & 1969 & O-90-92 \\
\hline Szene für neuen & 1969 & O-93 \\
\hline Begegnungen & 1970 & O-98 \\
\hline Si la carta dice: "Me gustaría volver a ver a Blanca" & 1970 & O-99 \\
\hline Sonora & 1970 & O-100 \\
\hline Arcanos & 1971 & O-102 \\
\hline Konstellation II & 1971 & O-103 \\
\hline Trigon & 1971 & O-104 \\
\hline Monodrama & 1971 & O-105-111 \\
\hline Kolot-Voices & 1971 & O-112 \\
\hline Canticas & 1971 & O-113 \\
\hline Annabel Lee & 1971 & O-114 \\
\hline Etüde & 1972 & O-117 \\
\hline Acting & 1972 & O-118 \\
\hline Actions for Piano & 1972 & O-119 \\
\hline An Anna Blume & 1972 & O-120 \\
\hline He engraves Signs into the Heavenly Sphere, & 1972 & O-122 \\
\hline Hommage to Picasso, & 1972 & O-123 \\
\hline Vera la morte & 1972 & O-124 \\
\hline Tetralog & $1972-74$ & O-125 \\
\hline Música para piano y vientos (Premonitions) & 1972 & O-126 \\
\hline Música para piano y cuerdas (Night Music) & 1972 & O-127 \\
\hline Música para piano y percusión (Dialogue) & 1974 & O-128 \\
\hline Música para piano y voz & 1974 & O-129 \\
\hline Signals & 1973 & O-130 \\
\hline Ballade for Voice and Irish Minstrel Harp & 1973 & O-133 \\
\hline De profundis & 1973 & O-134 \\
\hline Momentum & 1973 & O-135 \\
\hline Masada, & 1973 & O-136-142 \\
\hline Ritual, & 1974 & O-144 \\
\hline Ich komme & 1975 & O-154 \\
\hline$D A D A Y A$ mas $O N G$ & 1975 & O-162 \\
\hline Misa Sine Nomine & 1975-76 & O-146 \\
\hline Bereschít bará elohím et haschamáim weét haáretz & 1976 & O-147 \\
\hline Kyrie eleison & 1976 & O-148 \\
\hline Lied & 1976 & O-149 \\
\hline Gloria & 1976 & O-150 \\
\hline Chile & 1976 & O-151 \\
\hline Credo & 1976 & O-152 \\
\hline Benedictus & 1976 & O-153 \\
\hline Dona nobis pacem & 1976 & O-155 \\
\hline Babel & 1976 & O-156 \\
\hline Epilog & 1976 & O-157 \\
\hline Golem (7 partes) & 1976 & O-164-170 \\
\hline Aria & 1976 & O-171 \\
\hline Chanson & 1976 & O-172 \\
\hline
\end{tabular}

1 Respecto de su gráfica musical se recomienda ver el siguiente libro: David Schidlowsky (editor), Musikalische Graphik-Graphic Music. León Schidlowsky (Berlín: WVB, 2011). Una edición corregida, actualizada y ampliada fue publicada en castellano un año después: David Schidlowsky (editor). León Schidlowsky. Gráfica musical (Santiago: Ril Editores, 2012). Reseñas por Álvaro Gallegos Marino en RMCh, LXVI/218 (julio-diciembre, 2012), p. 132 y por Josefina Correa Téllez en RMCh, LXVII/220 (julio-diciembre, 2013), pp. 118-119. 


\begin{tabular}{|c|c|c|}
\hline Gráfica-musical / Multimedia & Año & Número del catálogo \\
\hline Schatten & 1976 & O-173 \\
\hline Annabel Lee & 1977 & O-180 \\
\hline Sound Poem & 1977 & O-181 \\
\hline Palindrom & 1979 & O-187 \\
\hline Klagemauer & 1979 & O-188 \\
\hline Tod einer Schauspielerin & 1979 & O-189 \\
\hline Deutschland, ein Wintermärchen (siete partes) & 1979 & O-190-196 \\
\hline Der schwarze Gott (siete partes), & 1980 & O-197-203 \\
\hline Quintet & 1980 & O-208 \\
\hline Nada & 1980 & O-209 \\
\hline Gilgul & 1980 & O-210 \\
\hline Für Klavier & 1980 & O-211 \\
\hline If we die (Aria) & 1981 & O-212 \\
\hline Yizkor & 1982 & O-214 \\
\hline Voices & 1982 & O-216 \\
\hline Motu propio & 1983 & O-224 \\
\hline Toccata & 1983 & O-225 \\
\hline Choral & 1984 & O-228 \\
\hline Prelude & 1984 & O-229 \\
\hline A Man between Stones & 1987 & O-236 \\
\hline Am Grab Kafka's & 1994 & O-262 \\
\hline Greise sind die Sterne geworden - eine moderne Passion & 1997 & O-270-80 \\
\hline Permutaciones & 2012 & O-369 \\
\hline Música incidental para teatro y cine & Año & Número del catálogo \\
\hline El mendigo & 1955 & O-20 \\
\hline Los vecinos & 1955 & O-21 \\
\hline Tiempo-vida & 1956 & $\mathrm{O}-22$ \\
\hline Música para pantomima & 1956 & O-25 \\
\hline El diablo y el ángel & 1956 & $\mathrm{O}-26$ \\
\hline Música para film & 1957 & O-39 \\
\hline Dulce Patria & 1959 & $\mathrm{O}-44$ \\
\hline La ciencia universitaria y la industria & 1959 & $\mathrm{O}-45$ \\
\hline Tierra dulce & 1959 & $\mathrm{O}-46$ \\
\hline Yizkor & 1982 & O-214 \\
\hline
\end{tabular}

\section{B. LISTADO CRONOLÓGICO DE OBRAS}

Para cada obra se señalan los siguientes antecedentes:

a - Título de la obra. Entre paréntesis, sus movimientos, cuando los tiene.

b - Año de composición.

c-Medio (ver abreviaturas).

d - Duración aproximada (abreviado Dur).

e - Autor del texto (abreviado Text).

f - Año de estreno, lugar e intérpretes (abreviado Estr). De acuerdo con la información disponible, se indican los estrenos de obras en países diferentes al país en que se efectuó el primer estreno. También se agregan selectivamente aquellas presentaciones en Chile que constituyen verdaderos reestrenos en el país, de obras cuyo estreno se efectuó hace muchos años. Estas se incluyen a continuación de la abreviatura (Reestr)

g - Editor, año de edición (abreviado $E d$ ),

h - Fonograma editado indicando tipo, título, intérpretes, institución editora, país y año u otro registro sonoro cuando no hay edición fonográfica (Fon).

i - Observaciones: dedicatorias, premios, lugar de composición, epígrafes, etc. (abreviado Obs:). 


\section{Abreviaturas}

A

ANC

arm

arp

B

b

Bar

bat

ca

camp

camp tu

cb

cdas

cel

$\mathrm{cfg}$

ci

$\mathrm{cl}$

clb

co

cofem

coinf

comasc

comx

conj

cor

ct

CT

cto

elect

Estr

fem

fg

fl

fln

Fon

gui

IEM

inst

mand

mar

mari

masc

Mez

mx

narr

ob

org

orq

orq ca

$\mathrm{OSCH}$

pand

perc

pf

picc

rec contralto

Asociación Nacional de Compositores, Chile armonio

arpa

bajo (voz)

bajo (a)

barítono

batería

cámara

campana (s)

campana tubular

contrabajo

cuerdas

celesta

contrafagot

corno inglés

clarinete

clarinete bajo

coro

coro femenino

coro infantil

coro masculino

coro mixto

conjunto

corno francés

cinta magnética

contratenor

cuarteto

eléctrica (o)

estreno

femenina

fagot

flauta

flautín

fonograma

guitarra

Instituto de Extensión Musical

instrumental

mandolina

maracas

marimba

masculino

mezzosoprano

mixtos

narrador

oboe

órgano

orquesta

orquesta de cámara

Orquesta Sinfónica de Chile

pandero

percusión (es)

piano

piccolo

recitante 
Abreviaturas

$\begin{array}{ll}\text { S } & \text { soprano } \\ \text { sax } & \text { saxofón } \\ \text { sol } & \text { solista (s) } \\ \text { T } & \text { tenor } \\ \text { tamb } & \text { tambor } \\ \text { tbn } & \text { trombón } \\ \text { timp } & \text { timbales } \\ \text { tpt } & \text { trompeta } \\ \text { tu } & \text { tuba } \\ \text { V, VV } & \text { voz, voces } \\ \text { va } & \text { viola } \\ \text { vc } & \text { violonchelo } \\ \text { vibr } & \text { vibráfono } \\ \text { vn } & \text { violín } \\ \text { xil } & \text { xilófono }\end{array}$

\section{Editoriales}

ANC. Asociación Nacional de Compositores. Chile

Ariadne Musikverlag, Viena, Austria

IMI. Israel Music Institute, Tel Aviv, Israel

Union Panamericana (Estados Unidos)

Schotts Soehne, Mainz (Alemania)

[O-1] Música para mi mamá, 1943, pf, Dur: 1',5”, Obs: música sin partitura, grabado en un disco privado 78, extraviado, Chile.

[O-2] Apología hebrea (3 partes en un movimiento), 1947/8, pf, Dur: 12', Obs: música sin partitura, grabado en un disco privado 78 (para Olga Licht), Chile.

[O-3] Olga, 1948, trozo sin partitura, pf, Dur: 3', Obs: grabado en disco privado 52 (para Olga Licht), Chile.

[0-4] Cinco poemas para contralto y piano (Rosa muerta, Jarro de angustia, Comienzo de otoño, Y álzate, Tiempo), 1952, A, pf, Dur: 4', Text: Cinna Lomnitz, Clara Drullinsky, compositor, Obs: cinco cortos poemas, "A mi hermana muerta", Chile.

[O-5] Tres trozos, 1952, pf, Dur: 3', Estr: Instituto Chileno-Británico de Cultura, Recital de Música Contemporánea, Agrupación Tonus, León Schidlowsky (piano), Santiago de Chile, 25 de julio de 1955. Lausitzer Kirche, Susanne Schidlowsky (piano), Berlín, 28 de febrero de 1999, Ed: Álbum para piano, IMI 392, Obs: "Ketty Bravo, Martínez Bonati, homenaje a Van Gogh, homenaje a Paul Klee", Chile.

[O-6] Seis miniaturas (Casa de noche, ¿Qué falta?, Come de la mano, El espíritu del teatro, ¿De donde, donde, hacia donde?, Fiesta oriental), 1952, pf, Dur: 7’ Estr: Instituto Chileno-Británico de Cultura, Recital de Música Contemporánea, Agrupación Tonus, León Schidlowsky (piano), Santiago de Chile, 25 de julio de 1955, Ed: Álbum para piano, IMI 392, Obs: Homenaje a Paul Klee, ilustraciones de seis reproducciones de Paul Klee, Chile.

[O-7] Elegía, 1952, cl o fl, cto cdas, Dur: 5', Estr: Instituto Chileno-Británico de Cultura, Recital de Música Contemporánea, Agrupación Tonus, Esteban Eitler (flauta), Cuarteto Santiago, Stefan Tertz y Ubaldo Grazioli (violín I y II), Raúl Martínez (viola), Hans Loewe (violonchelo), Santiago de Chile, 25 de octubre de 1957, Obs: Dedicada a Rodrigo Martínez, Chile.

[O-8] Zwei Lieder vom Tode (Dos canciones de la muerte) (O Herr gib jedem seine eigenen Tod, Schlusstuck), 1952, V, pf, Dur: 2', Text: Rainer Maria Rilke, Estr: Sala Mozart, Hanns Stein (Tenor), León Schidlowsky (piano), Santiago de Chile, 21 de agosto de 1957, Ed: Album for Voice and Piano, IMI 405, Chile.

[O-9] Drei Liebeslieder (Tres canciones de amor) (Nachts, Deine Lieder, Wenn es Abend wird), 1953, V, pf, Dur: 3', Text: Georg Trakl, Estr: Sala Mozart, Hanns Stein (Tenor), León Schidlowsky (piano), 
Santiago de Chile, 21 de agosto de 1957. Concierto en Homenaje al compositor en sus 75 años, Uta Buchheister (Voz), Martin Schneving (piano), Berlín, 26 de septiembre de 2006, Ed: Album for Voice and Piano, IMI 405, Obs: "Meiner Frau, zu unserem Hochzeitstag" (a mi esposa, en nuestro día de bodas), Alemania.

[O-10] Tres canciones bíblicas (Schema Yisroel, Hodu Ladonoi, Hallalujoh), 1954, S, fl, cl, vc, Dur: 3', Text: Biblia, Estr: Décimo Festival de Música Chilena, Grupo IEM. Santiago de Chile, 1966, Obs: Alemania.

[O-11] Dos Salmos, 1954, A, cl, clb, vn, vc, Text: Georg Trakl, Estr: Octavo Festival de Música Chilena (Mención honrosa), Música de Cámara, Primer Concierto de Selección, Ivonne Herbos (contralto), Rafael de Giudice (clarinete), Orlando Gutiérrez (clarinete bajo), Stefan Tertz (violín), Hans Loewe (violonchelo), Victor Tevah (director), Santiago de Chile, 22 de noviembre de 1962, Obs: "Meiner Frau gewidmet" (dedicado a mi esposa), "Detmold 27.6.1954", Alemania.

[O-12] Salmo, 1954, A, vn, cl, vc, fg, Dur: 5, Text: Georg Trakl, Obs: "Für Susanne Schidlowsky" (para Susanne Schidlowsky), Detmold, Alemania.

[O-13] Requiem (auf den Tod eines Knaben) (a la muerte de un muchacho), 1954, S, fl, cl, clb, tpt, 2 cor, arp, cel, pf, mand, xil, tamb, pand, timp, 8 vn, 4 va, 4 vc, Dur: 12, Text: Rainer Maria Rilke, Obs: "Primera obra sinfónica", "Detmold - 11 de octubre de 1954", Rainer Maria Rilke, Alemania.

[O-14] Fünf Kompositionen (Cinco composiciones), 1954, S, pf, Dur: 7', Text: Paul Klee, Morgenstern, Miguel Angel, Kandinsky, compositor, Ed: Album for voice and piano, IMI 405, Obs: Alemania.

[O-15] Sechs japanische Lieder (Seis canciones japonesas), 1954, Dur: 15', S, cl, clb, tpt, cor, arp, pf, vn, va, vc, Text: Haiku, Obs: "Dem Gedenken Gustav Mahler" (a la memoria de Gustav Mahler), Alemania.

[O-16] Trío (I movimiento, II movimiento: variaciones, III: Variaciones), 1955, fl, vc, pf, Dur: 7', Estr: Instituto Chileno-Británico de Cultura, Recital de Música Contemporánea, Agrupación Tonus, Esteban Eitler (flauta), Hans Loewe (violonchelo), Fré Focke (piano), Santiago de Chile, 25 de julio de 1955, Ed: IMI 7635 (22 de diciembre de 2005), Obs: "In memoriam Anton Webern en el décimo aniversario de su muerte, respetuosamente a Free Focke, Esteban Eitler, Hans Loewe”, Chile.

[O-17] Tres canciones (también titulada Tres poemas), 1955, Mez, pf, Dur:5', Text: Federico García Lorca, Vicente Huidobro, Estr: Instituto Chileno Alemán de Cultura, Ivonne Herbos (mezzosoprano), Rudolph Lehmann (piano), Santiago de Chile, 1964, Ed: Album for voice and piano, IMI 405, Obs: "A la memoria de Alban Berg", Chile.

[O-18] Tres canciones, 1955, S, fl, clb, vc, Dur: 3', Text: Antonio Machado, Estr: Décimo Festival de Música Chilena, Grupo IEM, Antonio Tauriello (director), 1966, Obs: Chile.

[O-19] Ocho estructuras, 1955, pf, Dur: 10', Estr: Salón Sur, Hotel Carrera, León Schidlowsky (piano), Santiago de Chile, 16 de agosto de 1955, Ed: Álbum para piano, IMI 392, Obs: Chile.

[O-20] El mendigo, 1955, fln, vn, ob, cl, perc (4 percusionistas), Dur: 15', Estr: Santiago de Chile, 15-30 de noviembre de 1955, Obs: Para el grupo de mimos de Enrique Noisvander. Obra presentada en el Teatro Cariola, partitura original y cinta magnética extraviadas, Chile.

[O-21] Los vecinos, 1955, orq ca, Dur: 10', Obs: Para el grupo de mimos de Enrique Noisvander. Obra presentada en el Teatro Cariola, partitura original extraviada, Chile.

[O-22] Tiempo-vida, 1956, ct, Estr: Santiago de Chile, 21 de agosto de 1957, Obs: Primer ensayo de música concreta, grabación en cinta magnética, para pantomimas, Chile.

[O-23] Música 1956 (2 movimientos), 1956, fl, ob, va, vc, Dur: 10', Estr: Instituto Chileno-Británico de Cultura, recital a cargo de la Agrupación Tonus, Esteban Eitler (flauta), Hans Loewe (oboe y violonchelo), Raúl Martínez (viola), Santiago de Chile, 21 de junio de 1956, Obs: Chile.

[O-24] Nacimiento, 1956, Dur: 3',10”, ct, Estr: Santiago de Chile, 15-30 de julio de 1957. Presentado en el Festival Synthése 06, segundo concierto, Rappel Historique, Bourges, Francia, 6 de junio de 2006, Obs: "Música concreta para pantomima" (música incidental para teatro de mimos), grabación efectuada en casa del compositor Fernando García, Chile. 
[O-25] Música para pantomima, 1956, tres percusionistas, 6 instrumentos, Estr: Santiago de Chile, julio 1956, Obs: Chile.

[O-26] El diablo y el ángel, 1956, Dur: 7’, Estr: Santiago de Chile, 21 de julio de 1957, Obs: Toccata para percusiones, música para pantomimas, cinta magnética extraviada de la versión del Grupo Tonus, Obs: Chile.

[O-27] Altazor VII Canto, 1956, rec, perc (2 percusionistas), Dur: 7’, Estr: Sala Exposición del Círculo de Periodistas, Casa Central Universidad de Chile, León Schidlowsky (recitante), Agrupación Tonus (percusionistas), Santiago de Chile, 25 de septiembre de 1956, Reestr: Pontificia Universidad Católica de Chile, Festival de Música Chilena Contemporánea, Aula Magna del Centro de Extensión de la Universidad Católica, Gerardo Urrutia (recitante), Grupo de Percusión de la UC (Sergio González, Gerardo Salazar, Álvaro Cruz, Héctor Mora, Frano Kovac, José Díaz), Carlos Vera (director), Santiago de Chile, 8 de septiembre de 1990, Obs: Partitura original extraviada, copia de la partitura en la biblioteca del Instituto de Música de la Pontificia Universidad Católica de Chile, Chile.

[O-28] Cuarteto mixto $\mathrm{N}^{\mathrm{o}}$ 1, 1956, fl, cl, vn, vc, Dur: 1', Estr: Instituto Chileno-Británico de Cultura, Recital de Música Contemporánea a cargo del Grupo Tonus, Esteban Eitler (flauta), Rodrigo Martínez (clarinete), Stefan Tertz (violín), Hans Loewe (violonchelo), Santiago de Chile, 25 de octubre de 1956, Obs: "Dedicado a la Agrupacion Tonus, de Santiago de Chile, fundada en 1952 por Free Focke y Esteban Eitler e integrada además por Hans Loewe, Rodrigo Martínez, Hans Karpisek, Magda Oetvoes, Raúl Martínez", Chile.

[O-29] Cuarteto mixto No 2, 1956, fl, ob, va, vc, Dur: indeterminada, Estr. Grupo Tonus, Santiago de Chile, 21 de junio de 1956, Obs: Chile.

[O-30] Cuatro epigramas, 1956, fl, pf, Dur: 2', Estr: Instituto Chileno-Británico de Cultura, Recital de Música Contemporánea a cargo de la Agrupación Tonus, (estrenado bajo el título Miniaturas para flauta y piano), Esteban Eitler (flauta), Free Focke (piano), Santiago de Chile, 4 de septiembre de 1956, Obs: "A Esteban Eitler", Santiago de Chile, 25 de junio de 1956.

[O-31] Musica para ballet infantil, 1956, 9 instrumentos de perc (tres percusionistas), Dur: indeterminada, Estr: Agrupación Tonus, Santiago de Chile, 16 de diciembre de 1956, Obs: Santiago de Chile, "A mis hijos David y Elias".

[O-32] Cinco piezas, 1956, pf, Dur: 6', Ed: Álbum para piano, IMI 392, Estr: (estrenada bajo el título 5 trozos para piano) Instituto Chileno-Británico de Cultura, Recital de Música Contemporánea, Agrupación Tonus, León Schidlowsky (piano), Santiago de Chile, 28 de septiembre de 1956. Tercer Programa de la BBC, Malcolm Troup (piano), Londres, 4 de marzo de 1963, Obs: "Para mi amigo Esteban Eitler", Chile.

[O-33] Concierto para seis instrumentos, 1957, sax, tpt, clb, pf, timp, xil, Dur: 10', Estr: VII Festival de Música Chilena, Concierto de Selección de Música de Cámara, y Concierto de Premios de Música de Cámara, Juan García (clarinete bajo), Jorge O’Kington (trompeta), Orlando Gutiérrez (clarón), Cirilo Vila (piano), Ramón Hurtado (xilofóno), Jorge Canelo (timbales), Agustín Cullell (director), Santiago de Chile, 7, 8 de diciembre de 1960. Centro Latinoamericano de Música, Universidad de Indiana, Quinto Festival de Primavera, Indiana University Philharmonic, Juan Pablo Izquierdo (director), Bloomington, Estados Unidos, 11 de mayo de 1967. Presentado en el II Festival de América y España, Madrid, 14 al 28 de octubre, 1967, Reestr: interpretada (bajo el nombre de Sexteto) en el segundo concierto del IV Festival de Música Chilena Contemporánea, organizado por el Instituto de Música de la Pontificia Universidad Católica de Chile, realizado en el Aula Magna de dicha Universidad en noviembre de 1993, por Luis Alberto Latorre (piano), Ricardo Vivanco (xilófono), Carlos Vera (timbales), Francisco Gouet (clarinete), Jeanette Lemus (clarinete bajo), Javier Contreras (trompeta), bajo la dirección de Alejandro Guarello, Ed: Union Panamericana (1964), Fon: Asociación Nacional de Compositores de Chile (ANC). Cinco Compositores chilenos. Conjunto Instrumental del I.E.M., Juan Pablo Izquierdo (director). Santiago de Chile, Odeón, 1967, Obs: "A mi esposa", "Segundo premio en el VII Festival de Música Chilena, 1960”, Chile. 
[O-34] Cuatro miniaturas (para cuarteto de vientos, I, II, III, IV), 1957, fl, ob, cl, fg, Dur: 10', Estr: Instituto Chileno-Británico de Cultura, Concierto de la Agrupación Tonus, Heriberto Bustamante (flauta), Hans Loewe (oboe), Rodrigo Martínez (clarinete), Hans Karpisek (fagot), Santiago de Chile, 25 de junio de 1959. Centro Latinoamericano de Música, Universidad de Indiana, Cuarto Festival de Primavera, Quintet of the Americas, Bloomington, 7 de mayo de 1966, Obs: Chile.

[O-35] In memoriam, 1957, vc, perc, Dur: 7’, Estr: Instituto Chileno-Británico de Cultura, concierto de la Agrupación Tonus, Inés Lobos (violonchelo), Jorge Canelo (percusión), Santiago de Chile, 31 de julio de 1958, Obs: Partitura original extraviada, Chile.

[O-36] Canciones de amor, 1957, narr, comx, Dur: 5', Text: Pierre Louÿs, Obs: Partitura original extraviada, Chile.

[O-37] Cantata negra (Llora Udeyandeya, Canción fúnebre, Ha bebido el dzan), 1957, T, pf, perc, Dur: 10', Text: Blaise Cendrars, Estr: Pontificia Universidad Católica de Chile, Sexto Concierto de la Temporada de 1957, "Autores chilenos Contemporáneos", conjunto ad-hoc, Raúl Rivera (director), Santiago de Chile, 21 de noviembre de 1957. Centro de Música Latinoamericana, Universidad de Indiana, Bloomington, 4 de marzo de 1963, Obs: Premio por Obra 1958, Chile.

[O-38] Cinco canciones de amor (Canción, noche, soledad, recuerdo, epitafio), 1957, T, fl, ob, va, vc, pf, Dur: 14', Text: anónimo, Estr: Instituto Chileno-Británico de Cultura, Recital de Música Contemporánea, Agrupación Tonus, Abelardo Quinteros (tenor), Clara Fries (flauta), Hans Loewe (oboe), Inés Lobos (violonchelo), Raúl Martínez (viola), Susanne Schidlowsky (piano), Santiago de Chile, 28 de noviembre de 1957, Obs: Chile.

[O-39] Música para film, 1957, ob, cl, va, vc, 2 perc (9 percusiones), Dur: 20', Estr: Temporada de Conciertos, Departamento de Música y Sonología, Facultad de Artes de la Universidad de Chile, Sala Isidora Zegers, Ensamble Compañía de Música Contemporánea, Carlos Valenzuela Ramos (director), Santiago de Chile, 29 de julio de 2015, Obs: Chile.

[O-40] Oda a la tierra, 1958, T y Bar (recitante), orq: 3 fl, 3 ob, 3 cl, 3 fg, 3 tpt, 4 cor, 3 tbn, tu, arp, pf, cel, xil, vibr, glockenspiel, timp, bat (6 percusionistas), cdas, Dur: 10-12', Text: versión libre del Génesis, Estr: VII Festival de Música Chilena, Segundo Concierto Sinfónico de Selección y Concierto Sinfónico de Premios, Hernán Würth (tenor), Manuel Cuadros (barítono recitante), OSCH, Jacques Bodmer (director), Santiago de Chile, 11 y 12 de diciembre de 1960, Obs: Chile.

[O-41] Caupolicán (Toqui Caupolicán, La guerra patria, El empalado, Epílogo), 1958, narrador (Bar), comx, 2 pf, cel, perc (7 percusionistas), Dur: 17', Text: Pablo Neruda, Estr: VI Festival de Música Chilena (Mención Honrosa), Manuel Cuadros (barítono recitante), Agrupación coral, Marco Dusi (director), Susanne Schidlowsky (piano), Cirilo Vila (piano), miembros de la Orquesta Sinfónica (percusión), Héctor Carvajal (director), Santiago de Chile, 28 de noviembre de 1958, Obs: Subtitulado "Relato épico", Chile.

[O-42] In memoriam (I-II-III), 1958, vc y pf, Dur: 7', Estr: Juventudes Musicales Chilenas, $5^{\text {a }}$ Temporada de Música de Cámara, Arnaldo Fuentes (violonchelo), Oscar Gacitúa (piano), Santiago de Chile, 18 de agosto de 1964, Obs: "A la memoria de mi padre, 12-VIII-1958", Chile.

[O-43] Isla Negra, 1959, fl, Dur: 5', Estr: X Festival de Música Chilena, Segundo Concierto de Cámara de Selección, Guillermo Bravo (flauta), Santiago de Chile, 7 de diciembre de 1966. Concierto en Homenaje al compositor en sus 75 años, Andrea Welte (flauta), Berlín, 16 de septiembre de 2006, Reestr: XV Festival Internacional de Música Contemporánea 2015, Concierto Inaugural en Homenaje al compositor, Sala Isidora Zegers, Departamento de Música y Sonología, Facultad de Artes de la Universidad de Chile, Wilson Padilla (flauta), Santiago de Chile, 12 de enero de 2015, Ed: IMI 478 (1 de enero de 1976), Obs: Subtitulada "Invención para flauta", "dedicada a Esteban Eitler", Chile.

[O-44] Dulce Patria, 1959, música incidental para cine (director cinematográfico Fernando Balmaceda), Dur: 10', Obs: En coautoría con Fernando García, partitura original extraviada, Chile. 
[O-45] La ciencia universitaria y la industria, 1959, música incidental para cine (director cinematográfico Fernando Balmaceda), Dur: 20', Obs: En coautoría con Fernando García, partitura original extraviada, grabada en la Universidad de Chile, Chile.

[O-46] Tierra dulce, 1959, música incidental para cine, Dur: 2', Estr: Temporada Fílmica, Santiago de Chile, 1959, Obs: En colaboración con Fernando García, Chile.

[O-47] Epitafio a Vicente Huidobro, 1959, S, fl, xil, vibr, 1 perc (3 percusiones), Dur: 10', Text: Vicente Huidobro, Estr: Pontificia Universidad Católica de Chile, Instituto de Música, V Festival de Música Chilena Contemporánea, Aula Magna del Centro de Extensión, Paula Elgueta (Soprano), Wilson Padilla (flauta), José Díaz (xilófono), Marcelo Espíndola (vibráfono), Sergio Menares, Gonzalo Muga (percusión), Carlos Vera (director), Santiago de Chile, 20 de noviembre de 1995. Obs: Chile.

[O-48] Tríptico (I-II-III), 1959, orq: 2 fl, 2 cl, 2 fg, cfg, 3 tpt, 4 corn, 3 tbn, tu b, xil, vibr, 2 mar, perc (7 percusionistas), vn I, vn II, va, vc, cb, Dur: 7', Estr: OSCH, Temporada de Primavera, Teatro Alameda, Agustín Cullell (director), Santiago de Chile, 30 de septiembre de 1960. Ed: IMI 265 (15 de mayo de 1973), Fon: León Schidlowsky, Obras Sinfónicas. CD. Compositores chilenos. Volumen 4. Santiago de Chile, ABA SVR 17000-4, 2013. Reseña del fonograma por Fernando García en RMCh, LXVIII/221 (enero-junio, 2014), pp. 102-106, Obs: "A la memoria de Roberto Falabella Correa”, Isla Negra "21-VII-1959", Primer Premio del Concurso CRAV (Compañía Refinería de Azúcar de Viña del Mar), 1963, Chile.

[0-49] Cadencia, 1961, vc, Dur: 2', Estr: $7^{\text {a }}$ Temporada oficial, Ciclo Latinoamericano, $6^{\circ}$ Concierto de abono, Hans Loewe (violonchelo), Orquesta Filarmónica de Chile, Guillermo Espinoza (director), Santiago de Chile, 1961, Obs: Cadencia para el Concierto para violonchelo y orquesta de Robert Schumann, partitura original extraviada, Chile.

[O-50] La noche de cristal (I Preludio, II Meditación, III Catástrofe, IV Plegaria), 1961, T, comasc, orq: 2 fl, fl picc, $2 \mathrm{ob}$, ci, clb, $2 \mathrm{fg}$, cfg, 4 tpt, 4 cor, 3 tbn, tu, 2 arp, cel, vn I, vn II, va, vc, cb, perc (5 percusionistas), Dur: 14', Text: fragmentos de un rezo judío tradicional, Estr: Orquesta Filarmónica de Chile, $8^{\mathrm{a}}$ Temporada Oficial, $6^{\circ}$ Concierto de Abono, Hanns Stein (Tenor), Juan Matteucci (director), Santiago de Chile, 31 de mayo de 1962. Orquesta Nacional de México, Luis Herrera de la Fuente (director), Ciudad de México, 12 de julio de 1963, Reestr: Temporada 2015, Centro de Extensión Artística y Cultural de la Universidad de Chile, Concierto $\mathrm{N}^{\circ}$ 5, Del Barroco a la Modernidad, Teatro Universidad de Chile, Camerata Vocal de la Universidad de Chile, Orquesta Sinfónica de Chile, Juan Pablo Izquierdo (director), 25 y 26 de abril de 2015, Ed: IMI 209 (1 de abril de 1971), Fon: León Schidlowsky, Obras Sinfónicas. CD. Compositores chilenos. Volumen 4. Santiago de Chile, ABA SVR 17000-4, 2013. Reseña del fonograma por Fernando García en RMCh, LXVIII/221 (enerojunio, 2014), pp. 102-106, Obs: "Por estos yo lloro ... En recuerdo de los mártires de nuestro tiempo... Perecidos por ser judíos”, Premio Olga Cohen 1962, Premio por Obra 1963. Chile. Partitura original extraviada. Esta obra sirvió de base al ballet Inmolación sobre una coreografía de Germán Silva, escenografía de Emilio Hermansen y trajes de Edith del Campo, estrenado por el Ballet Nacional Chileno en el Teatro Municipal de Santiago el 6 de junio de 1965.

[O-51] Soliloquios, 1961, cl, arp, gui, vc, pf, cel, vibr, perc (3 percusionistas), Dur: 10', Estr: Universidad Católica de Chile, Juan Correa (clarinete), Arlette Bezdecki (arpa), Luis López (guitarra), Hans Loewe (violonchelo), Galvarino Mendoza (piano, celesta), Ramón Hurtado, Jorge Canelo y Uldaricio Oñate (percusión), Juan Pablo Izquierdo (director), Santiago de Chile, 23 de julio de 1962. Centro Latinoamericano de Altos Estudios Musicales, Tercer Festival de Música Contemporánea, Segundo concierto, Buenos Aires, R. Argentina, 15 al 18 de octubre de 1964, Ed: IMI (1 de enero de 1975), Obs: "Por encargo de Carlos Riesco", "dedicada a Juan Pablo Izquierdo", Chile.

[O-52] Zwei Lieder (Dos canciones), 1962, T, arp, cel, vibr, perc (2 percusionistas), Dur: 7', Text: Georg Trakl, Estr: VIII Festival de Música Chilena (Mención Honrosa), Música de Cámara, Segundo Concierto de Selección, Hernán Würth (Tenor), Arlette Bezdechi (arpa), Hilda Cabezas (celesta), Iris Sangüesa y Jorge Canelo (percusión), Victor Tevah (director), Santiago de Chile, 24 de noviembre de 1962, Ed: IMI 266 (1 de abril de 1974), Obs: "Für Hernan Wurth" (para Hernán Würth), Chile. 
[O-53] Eróstrato, 1962, pf, ondas Martenot y 40 perc (19 percusionistas), Dur: 10', Estr: Instituto de Extensión Musical, Temporada de Cámara, Santiago de Chile, 1964, Obs: inspirada en un poema de Gabriel D’Annunzio, "Santiago de Chile, agosto de 1962”, "Obra encargada por el III Festival Interamericano de Música de Washington", "Todos mis sentidos vigilaban, en la espera de la alegría Obscura. Una belleza indecible yo sentía expandirse por mis miembros como quien se transfigura. Morir o gozar! Gozar o morir!", Chile.

[O-54] Amatorias (preámbulo, coloquio, canción, interludio, horizonte, cadenza, epílogo), 1962-3, T, conj inst: sax alto, gui eléctrica, arp, cel, pf, vibr, xylorimba-camp, perc (2 percusionistas), Dur: 12', Text:Vicente Huidobro, Estr: IX Festival de Música Chilena (Segundo Premio), Tercer Concierto de Cámara de Selección, Hernán Würth (tenor), Conjunto instrumental, Juan Pablo Izquierdo (director), Santiago de Chile, 2 de diciembre de 1964, Obs: Premio Olga Cohen 1964, Chile.

[O-55] De profundis (Desde lo profundo), 1963, S, C, T, fl, ob, clb, sax alto, tpt, tbn, vn, va vc, Dur: 12', Text: de la Biblia (fragmentos del Salmo 129 en latín), Estr: X Festival de Música Chilena. Tercer Concierto de Cámara de Selección, Concierto Final de Cámara, Mary Ann Fones (soprano), Carmen Luisa Letelier (contralto), Hernán Würth (tenor), Guillermo Bravo (flauta), Enrique Peña (oboe), Orlando Gutiérrez (clarinete), Oscar Moya (saxofón alto), Pastor Gutiérrez (trompeta), Héctor Reyes (trombón), Jaime de la Jara (violín), Manuel Fuentes (viola), Arnaldo Fuentes (violonchelo), Antonio Tauriello (director), Santiago de Chile, 3 y 5 de enero de 1967, Obs: "(Obra comisionada por la Universidad Católica de Chile)", "In memoriam Daphne Sauré", Chile.

[O-56] Invocación, 1964, S, narr, orq: 12 vn I, 12 vn II, 10 va, 10 vc, 8 cb, cel, perc (5 percusionistas), Dur: 14', Text: compositor, Estr: IX Festival de Música Chilena (Segundo Premio), Concierto Sinfónico de Selección, Ivonne Herbos (soprano), Hanns Stein (narrador), OSCH, Agustín Cullell (director), Santiago de Chile, 20 de noviembre de 1964. Presentada en el II Festival de América y España, Madrid, 14 al 28 de octubre, 1967, Ed: IMI 264 (1 de abril de 1974), Fon: Grabada para un disco en 1965 en Leipzig, Alemania, Leipzig Radio Symphony Orchestra, Dirección: Herbert Kegel (por razones políticas en la RDA nunca se publicó el disco). León Schidlowsky. Obras Sinfónicas. CD. Compositores chilenos. Volumen 4. Santiago de Chile, ABA SVR 17000-4, 2013, Obs: "A Hermann Scherchen”, Chile. Reseña del fonograma por Fernando García en RMCh, LXVIII/221 (enerojunio, 2014), pp. 102-106.

[O-57] Amereida (Llaqui, Memento, Ecce Homo), 1965 - 1969, (cada una de las partes puede ser tocada por separado):

[O-58] 1: Llaqui (elegía para orquesta), 1965, narr, orq: 2 fl, 2 ob, 2 cl, 2 fg, 3 tpt, 3 cor, 2 tu, 3 tbn, 12 vn I, 12 vn II, 8 va, 8 vc, 8 cb, Dur: 10', Text: Javier Heraud, Estr: Orquesta des Rundfunks der Italienischen Schweiz (Orquesta de la Radio de la Suiza Italiana), Hermann Scherchen (director), Lugano, 12 de marzo de 1965. Teatro Municipal, decimotercer Concierto, Orquesta Filármonica de Chile, Juan Pablo Izquierdo (director), Santiago de Chile, 1966. Holanda, Orquesta Residentie, Juan Pablo Izquierdo (director), Rotterdam, 1967. Centro Latinoamericano de Música de Indiana, Sexto Festival de Primavera, Orquesta de Conciertos de la Universidad de Indiana, Juan Pablo Izquierdo (director), Bloomington, 19 de abril de 1968, Ed: IMI 269 (1 de abril de 1974), Fon: León Schidlowsky, Obras Sinfónicas. CD. Compositores chilenos. Volumen 4. Santiago de Chile, ABA SVR 17000-4, 2013, Obs: Dedicado "a la memoria del joven poeta peruano Javier Heraud", "Llaqui: palabra quechua que significa pena, tristeza”, Chile. Reseña del fonograma por Fernando García en RMCh, LXVIII/221 (enero-junio, 2014), pp. 102-106.

[O-59] 2: Memento (Meditación americana, Dies Irae, Palabra de guerrillero), 1967, S, orq: fl picc, 2 fl, 3 ob, 3 cl, 3 fg, 4 tpt, 4 cor, 4 tbn, xil, mari, perc (2 percusionistas), vn I, vn II, va, vc, cb, comisionada por Herman Scherchen, Dur: 10', Text: Javier Heraud, Estr: Catherine Gayer (soprano), Orquesta de la Radio Hessischer Rundfunk, Juan Pablo Izquierdo (director), Frankfurt, 21 de enero de 1972. Ed: IMI 226 (2 de enero de 1972), Obs: Dedicada a la memoria del sacerdote Camilo Torres Restrepo, Chile.

[O-60] 3: Ecce Homo (¡He aquí el hombre!), 1969, S, orq: 12 vn I, 12 vn II, 10 va, 10 vc, 8 cb, pf, perc (4 percusionistas), Dur: 9', Text: del compositor basado en fragmentos del diario del Che Guevara, Estr. Catherine Gayer (soprano), Orquesta de la Radio Hessischer Rundfunk, Juan Pablo 
Izquierdo (director), Frankfurt, 21 de enero de 1972, Ed: IMI 7882 (2 de diciembre de 2009), Obs: "A la memoria de Ernesto Guevara", Chile.

[O-61] Nueva York, 1965, orq: 2 fl, 3 ob, ci, 2 cl, 3 fg, cfg, 4 tpt, 5 cor, 3 tbn, tu, perc (4 percusionistas), 12 vn I, 12 vn II, 10 va, 10 vc, 8 cb, Dur: 10', Estr: III Festival de Música de Caracas, Concierto Inaugural, Orquesta Sinfónica de Venezuela, Gonzalo Castellanos (director), Caracas, 6 de mayo de 1966, XXV Temporada Oficial, Decimotercer Concierto, OSCH, Víctor Tevah (director), Santiago de Chile, 1966, Fon: Música chilena del siglo XX. CD. Volumen VI. Santiago de Chile, ANC-6003-6, Ministerio de Educación, FONDART (Fondo para el Arte y la Cultura), 2000, Fon: León Schidlowsky. Obras Sinfónicas. CD. Compositores chilenos. Volumen 4. Santiago de Chile, ABA SVR 17000-4, 2013, Reseña de este último fonograma por Fernando García en RMCh, LXVIII/221 (enero-junio, 2014), pp. 102-106, Ed: IMI 323 (1 de enero de 1975), Obs: Motto: "Nueva York / un triángulo escalero / asesina a un cobrador / El cobrador de hojalata / y el triángulo de prisa / otra vez a su pizarra / Nick Carter no entiende nada. / Oh Nueva York (De Rafael Alberti), Dedicada "A mis hermanos negros de Harlem", Comisión del Festival Interamericano de Música de Washington, Chile.

[O-62] Deutsches Tagebuch (Diario alemán) (An die Toten von Buchenwald, An meine Landsleute, An die Nachgeborenen), 1965, Bar (rec), comx, orq: fl picc, 2 fl, 3 ob, cl picc, 2 cl, clb, 1 tpt picc, 3 tpt, tpt b, 6 cor, 4 tbn, tu, pf, perc (5 percusionistas), 12 vn I, 12 vn II, 10 va, 10 vc, 8 cbs, Dur: 30', Text: Berthold Brecht, Obs: "An die Toten von Buchenwald", Nathan Notowitz gewidmet, "An meine Landsleute", für Martina Hemsler, "An die Nachgeborenen” zum Gedaechtnis an Hans Eisler, Stephi Eisler gewidmet" ("A los muertos de Buchenwald", dedicado a Nathan Notowics, "A mis compatriotas", para Martina Hemsler, "A los hombres futuros”, dedicado a Hans Eisler, Stephi Eisler), Chile.

[O-63] Three Hebrew Pieces (también titulada Tres coros hebreos) [1 - If I forget Thee, OJerusalén (Si yo me olvidare de ti, Jerusalén) (Salmo 137,5-6), 2 - Out of depths (Desde lo profundo), 3 - I believe (Yo creo) ], 1965-6, comx, Dur: 9', Text: Biblia, Estr: primer coro (If I forget Thee, OJerusalén), Temporada de Música de Cámara, $25^{\circ}$ aniversario del Instituto de Extensión Musical, Cuarto Concierto, Coro de Cámara de Valparaíso, Marco Dusi (director), Santiago de Chile, 14 de junio de 1965, Durante septiembre del mismo año este conjunto estrenó el primer coro en la gira efectuada por Washington, DC, Nueva York, Boston, Cornell en los Estados Unidos, además de Panamá y Lima, Perú. Ed: IMI 210 (1 de abril de 1971), Fon: Primer coro (If I forget Thee, O Jerusalén). Antología coral chilena. ANC-AC-7000-1, Santiago de Chile, Ministerio de Educación, FONDART (Fondo para el Arte y la Cultura), 2002, Obs: 1 coro "a mi hijo David", 2 coro "A mi hijo Elías, 3 coro "A mi hija Judith", Chile.

[O-64] Cuatro episodios (I-II-III-IV), 1966, rec masc y pf, Dur: 5', Text: “Titulares de diarios, declaraciones de hombres célebres, poetas usados en forma fragmentaria como Vicente Huidobro y Abraham Jesús Brito", Estr: Biblioteca Nacional, Fernando Torm, piano y voz, Santiago de Chile, 22 de diciembre de 1966, Obs: Chile.

[O-65] Tres versos del capitán (El alfarero, Siempre, Levántate conmigo), 1966, T, pf, cel, perc (4 percusionistas), Dur: 7', Text: Pablo Neruda, Estr. X Festival de Música Chilena, Primer Concierto de Cámara de Selección, Conciertos Finales de Cámara, Hernán Würth (Tenor), Hilda Cabezas (piano y celesta), Jorge Canelo, Guillermo Rifo, Uldaricio Oñate y Patricio Salazar (percusión), Antonio Tauriello (director), Santiago de Chile, 6 de diciembre de 1966 / 5 de enero de 1967, Obs: "Caracas 10 de junio de 1966", Chile.

[O-66] Monumento a Bolívar, 1966, narr, orq: 2 fl, 2 ob, 2 cl, 2 fg, 4 tpt, 4 cor, 3 tbn, 1 tu, perc (cinco percusionistas), pf, 12 vn I, 12 vn II, 10 va, 10 vc, 8 cb, co, Dur: 25', Text: Miguel Ángel Asturias, Pablo Neruda, Obs: "A Caracas en el Cuatricentenario de su fundación”, "18 de septiembre 1966", cita en la partitura: poema Credo, de Miguel Ángel Asturias, Chile.

[O-67] Jeremías, 1966, 2 S, 2 A, 2 T, 2 B, 4 vn I, 3 vn II, 3 va, 3 vc, 2 cb, Dur. 15', Text: Lamentaciones de Jeremías (fragmentos), Obs: "Esta obra fue comisionada en octubre de 1966 por el Departamento de Música de la Universidad Católica de Chile”, “5-XI-1966”, Chile. 
[O-68] Lamentación, 1966, comx, Dur: 7', Text: Jeremias 12:1, Estr. Coro de la Universidad de Chile, Santiago de Chile, 1966, Ed: IMI 211 (1 de abril de 1971), Obs: Obtuvo Premio ex aequo en el Concurso convocado por la Asociación Coral Chilena en 1967, en la categoría de composiciones corales para conjunto de adultos.

[O-69] Kaddish, 1966-7, vc, orq: 2 fl, 1 fl en Sol, 2 ob, ci, 2 cl, 1 clb, 2 fg, 1 cfg, 4 tpt, 4 cor, 3 tbn, 1 tu, cel, vibr, camp, 2 timp, 24 vn I, 10 va, 8 cb, Dur: 16', Estr: Concurso de Música CRAV [Compañía de Refineria de Azúcar de Viña del Mar en colaboración con el IEM y la ANC] (Primer Premio en la sección Obras Sinfónicas con Solista), Teatro Municipal, Eduardo Sienkewicz (violonchelo), OSCH, David Serendero (director), 16 de noviembre de 1967; repetición en la XXVII Temporada Oficial de la OSCH, Noveno Concierto, Eduardo Sienkiewicz (violonchelo), Juan Pablo Izquierdo (director), Santiago de Chile, 5 de julio de 1968, Ed: IMI 280 (24 de julio de 1973), Fon: León Schidlowsky. Obras Sinfónicas. CD. Compositores chilenos. Volumen 4. Santiago de Chile, ABA SVR 17000-4, 2013, Obs: Motto: Ecclesiastes, Ch. 3,22", "In memoriam Hans Loewe", "Santiago de Chile, 30-1-1967”, Chile. Reseña del fonograma por Fernando García en RMCh, LXVIII/221 (enero-junio, 2014), pp. 102-106.

[O-70] Imprecaciones (subtitulada Por las víctimas del Vietnam), 1967, coro (20 sopranos, 20 contraltos, 20 tenores, 20 bajos), Dur: 5', Estr: Cuba, Obs: "7-II-1967", Chile.

[O-71] Estudiante baleado, 1967, V, pf, Dur: 7', Text: Gonzalo Rojas, Estr: Fernando Torm (piano), Santiago de Chile, 1967, Obs: "A la memoria de Santiago O. Pampillon, estudiante argentino, 1941-1966, asesinado en Córdoba, República Argentina”, "14-II-1967”, Chile.

[O-72] Eclosión, 1967, rec, V fem, fl picc, tpt, pf (sin tapa), vibr, perc (3 percusionistas), 6 vn, 7 va, 8 vc, 9 cb, Dur: 7', Text: texto y foto pegada en la partitura de Julius Robert Oppenheimer, aclaración sobre la posición exacta de los instrumentos en el escenario, Estr: XXX Temporada de Invierno, OSCH, Juan Pablo Izquierdo (director), Santiago de Chile, 1971. Wolfgang Korb (recitante), Catherine Nick (V), Sprechgesang, Ensemble PanArte, Errico Fresis (director), Saarbruecken, 1 de marzo de 1998, Ed: IMI 208 (1 de abril de 1971), Obs: "24-II-1967”, Chile, obtuvo una Mención en el Segundo Concurso de Composición Musical, categoría A, Música de Cámara, Casa de las Américas, La Habana, Cuba, 1967.

[O-73] Epitafio para Hermann Scherchen, 1967, orq: 4 fl, 4 ob, 4 cl, 4 fg, 4 tpt, 4 cor, 4 tbn, perc (4 percusionistas), 12 vn I, 12 vn II, 10 va, 10 vc, 8 cb, Dur: 10', Estr: Concurso de Música CRAV [Compañía de Refineria de Azúcar de Viña del Mar en colaboración con el IEM y la ANC] (Segundo Premio en la sección de Obras Sinfónicas), Teatro Municipal, OSCH, David Serendero (director), Santiago de Chile, 16 de noviembre de 1967, Ed: IMI 322 (1 de enero de 1975), Obs: Cita de Hermann Scherchen: "Si la materia responde a la idea, hay infinitas posibilidades de una realización sonora", "24-IV-1967", Chile.

[O-74] Cuarteto de cuerdas, 1967, cto cdas, Dur: 15', Estr: The IV Inter-American Music Festival, The Beaux-Arts String Quartet, Washington, D.C., 24 de junio de 1968, Ed: Schotts Soehne in Mainz (21 de agosto de 1969), Obs: "Comisionado por el IV Inter-American Music Festival”, dedicación: "A mi hijo David en el año de su Bar Mitzva", "2-XII-1967”, Chile.

[O-75] Visiones, 1967, 7 vn, 2 va, 2 vc, 1 cb, Dur: 10', Estr: III Semana de Música Contemporánea, Departamento de Música de la Pontificia Universidad Católica de Chile, Universidad Católica de Valparaíso, IEM, ANC, 23 al 26 de enero de 1968, Orquesta de Cámara de la Pontificia Universidad Católica de Chile, Fernando Rosas (director), Presentada en Europa por la Orquesta de Cámara de la Pontificia Universidad Católica de Chile, bajo la dirección de Fernando Rosas, en una gira que se inició el 4 de enero de 1970, Reestr: $74^{\mathrm{a}}$ Temporada Artística, Universidad Técnica Federico Santa María, Orquesta de Cámara de Chile, Juan Pablo Izquierdo (director), Valparaíso, 11 de julio de 2015, Ed: IMI 463 (1 de diciembre de 1975), Fon: León Schidlowsky. Obras Sinfónicas. CD. Compositores chilenos. Volumen 4. Santiago de Chile, ABA SVR 17000-4, 2013, Obs: "Obra comisionada por el Departamento de Música de la Universidad Católica de Chile", "Dedicada a la Orquesta de Cámara de la Universidad Católica de Chile y a Fernando Rosas, su director", tiene como moto a Isaías 24,18, "24-XII-1967”, Chile. Reseña del fonograma por Fernando García en RMCh, LXVIII/221 (enero-junio, 2014), pp. 102-106. 
[O-76] Quinteto de vientos, 1968, fl, ob, cl, tpt francesa, cor, Dur: 8', Ed: IMI 281 (24 de julio de 1973), Fon: Israeli Works for Woodwind Quintet, New Israeli Woodwind Quintet, MII-CD-24, Tel Aviv 1999; CD, Santiago de Chile, Ministerio de Educación, Fondo para el Arte y la Cultura (FONDART), 2002, Obs: "In memoriam Juan Correa”, “5-I-1968”, Chile.

[O-77] Seis Hexáforos para Juan Manuel, 1968, perc (6 percusionistas), Dur: 7', Estr: Orquesta de Cámara de la Universidad Católica de Chile, Grupo Novarum de Percusión, Juan Pablo Izquierdo (director), Santiago de Chile, 14 de agosto de 1968, Presentada en el Séptimo Festival de Primavera del Centro de Música Latinoamericana de la Universidad de Indiana, Bloomington, realizado entre el 13 y el 15 de mayo de 1970. Ed: IMI 282 (24 de julio de 1973), Obs: "A la memoria de Juan Manuel Valcárcel, emigrado español que quiso echar su suerte con los pobres de la tierra”, "11-1-1968", Chile.

[O-78] Requiem para coro a cappella, 1968, comx, Dur: 20', Text: Misa de Difuntos, Estr: Schola Cantorum Stuttgart, Dirección C. Gotwald, Stuttgart, 1970. Ulteriormente, la obra ha sido interpretada en múltiples ocasiones en Alemania y en otros países. Ed: Schotts Soehne in Mainz (11 de marzo de 1970), Obs: "In memoriam Juan Agustin Peni”, “7-II-1968”, Chile.

[O-79] Espergesia, 1968, V, perc (6 percusionistas), Dur: 8', Text: César Vallejo, Estr: III Semana de Música Contemporánea, Universidad Católica de Chile, Juan Pablo Izquierdo (director), Santiago de Chile, 1968. Centro de Música Latinoamericana, Hernán Würth (tenor), I. U. Percussion Ensemble, Juan Pablo Izquierdo (director), Bloomington, Indiana, 16 de marzo de 1969, Obs: "Espergesia ha sido escrita para el Departamento de Música de la Universidad Católica de Valparaiso", "20VIII-1968", Chile.

[O-80] Interludio, 1968, gui de diez cdas, Dur: 10', Obs: "Para Narciso Yepes y su guitarra", "18 Oct. 1967 (sic)", Chile.

[O-81] Autorretrato del poeta, 1968, narr, perc (seis percusionistas), Dur: 9', Text: Pablo de Rokha, Obs: "24-XI-1968", Chile.

[O-82] Recital poético-musical, 1968, seis metrónomos, fl, ob, cl, fg, cor, cb, perc (3 percusionistas), Dur: 7', Obs: "Obra encargada por el Departamento de Música y para uso de la Vicerrectoría de Comunicaciones de la Universidad Católica”, partitura original extraviada, copia de la partitura en el archivo del compositor y en la biblioteca del Instituto de Música de la Pontificia Universidad Católica, Chile.

[O-83] Grabstein für Else Lasker-Schüler (Lápida para Else Lasker-Schüler) (Weltende, Abends, Ich liege wo am Wegrand urbermattet), 1969, V fem, ci, clb, cel, arm, vn, vc, perc (6 percusionistas), Dur: 7', Text: Else Lasker-Schüler, Obs: escritura proporcional, "Weltende" (Tel Aviv 26 de enero de 1969), "Abends" (Tel Aviv, 2 de febrero de 1969), "Ich liege wo am Wegrand urbermattet" (Tel Aviv 10 de febrero de 1969), Israel.

[O-84] Grabstein für Else Lasker-Schüler (Lápida para Else Lasker-Schüler), 1969, S, perc (6 percusionistas, Dur: 7', Text: Else Lasker-Schüler, Estr: Ralf Peter (contratenor), Ensemble Panarte, Errico Fresis (director), Saarbrücken, Alemania, 20 de diciembre de 1997, Ed: IMI 479 (1 de enero de 1976), Israel.

[O-85] Lyrik, 1969, S, perc (5 percusionistas), Dur: 5', Text: Lind Dsung-Yuan, Tang Yin, Li Pin (traducción al alemán por Gunther Debon y Werner Speiser), Ed: IMI 481 (1 de enero de 1976), Obs: escritura proporcional, Israel.

[O-86] Dialog mit Martin Buber (Diálogo con Martin Buber), 1969, comasc, vn, perc (5 percusionistas), Dur: 6', Text: Martin Buber (Der Fiedler), Obs: Escritura proporcional, "Lachen-Speyerdorf 1969", Israel.

[O-87] Eli Eli lama azavtani (Mein Gott, mein Gott, warum hast Du mich verlassen) (Oh Dios, Oh Dios, porque me has abandonado), 1969, 3 S, 3 A, 3 T, 3 B, Dur: 2', Text: de la Biblia [en hebreo], Obs: Escritura proporcional, Israel.

[O-88] Bereshit (En el principio), 1969, 3 S, 3 A, 3 T, 3 B, Text: Genesis 1,1-3, Ed: IMI 6566; Obs: Escritura proporcional, "Lachen-Speyerdorf 1969", Israel. 
[O-89] Konstellation I, 1969, orq cdas, Dur: abierta, Obs: Partitura gráfica, Israel.

[O-90-92] Sprüche (Proverbios), (3 partes), 1969, 12 solo de voces (6 S y 6 B), Dur: abierta, Obs: Cada una de las tres partes puede ser ejecutada por separado, partitura gráfica, Israel.

[O-93] Szene für neuen (Escena para lo nuevo), 1969, S, fl, cb, instr de perc, Estr New Dimensions in Music (Nuevas dimensiones en música, Tel Aviv 1969, Obs: Partitura gráfica, Isarel.

[O-94] Die Menschen (Los seres humanos), 1969, ópera en 5 actos, 6 S, 4 A, 2 Bar, 9 T,8 B, 2 comasc, 1 cofem, mimos, comx, 4 act, orq: 3 fl, 3 ob, 3 cl, 3 fg, 1 sax tenor, 4 tpt, 4 cor, 4 tbn, 1 tu, 6 vn, 6 va, 6 vc, 6 cb, perc (6 percusionistas), Dur: 100', Text: del compositor, según Walter Hasenclever, Obs: "Musikalisches Schauspiel in 5 Akten" (obra de teatro musical en 5 actos), Israel.

[O-95] Sexteto, 1970, fl, cl, pf, vn, vc, perc, Dur: 12', Estr: The Israel Chamber Ensemble, 14 de agosto de 1970, Ed: IMI 298 (1 de mayo de 1974), Obs: "To my sons Yuval and Noam", "Commissioned by the Composers workshop" (A mis hijos Yuval y Noam, encargado por el Taller de Compositores), Motto: cita de Amos 8:3, Israel.

[O-96] Babi Yar, 1970, pf, perc, 7 vn I, 5 vn II, 4 va, 3 vc, 3 cb, Dur: 15', Estr: Samuel Rubin Academy Chamber Orchestra, Shalom Ronly- Riklis (director), Tel Aviv,10 de febrero de 1971, Ed: IMI 189 (1 de febrero de 1971), Obs: "Tel Aviv XII-1970", "Those who want to see the light as it is must retire into the shadow. Shadow brighter than the sun: cool shadow of Freedom" (Aquellos que desean ver la luz como es se deben retirar en las sombras. La sombra es más brillante que el sol: la fría sombra de la libertad) from Hans Magnus Enzensberger: Shadow Realm" (El reino de las sombras), comisionada por el Aniversario de la Academia de Música Rubin de Tel Aviv, Israel.

[O-97] Serenata, 1970, 5 vn I, 4 vn II, 2 va, 3 vc, 3 cb, perc (1 percusionista), Dur: 10', Estr: Israel Chamber Ensamble, Gary Bertini (director), 26 de junio de 1971, Ed: IMI 190 (1/3/1971), Obs: "To Dalia Yadlin" (A Dalia Yadlin), "Com. by the Composer Fund Jerusalén, for the Israel Chamber Ensemble" (encargo del Fondo del Compositor en Jerusalén, para el Israel Chamber Ensemble), "Tel Aviv 11-1970", Israel.

[O-98] Begegnungen (Encuentros), 1970, pf, perc, Dur: abierta, Obs: Partitura gráfica, Israel.

[O-99] Si la carta dice: "Me gustaría volver a ver a Blanca", 1970, pf, perc, Dur: abierta, Text: anónimo, Obs: Partitura gráfica, Israel.

[O-100] Sonora, 1970, perc (1-4 percusionistas), Dur: abierta, Ed: IMI 267 (15 de mayo de 1973), Obs: Partitura gráfica, Israel.

[O-101] In memoriam (Azkarat neshamot), 1971, Bar, fl, ob cl, vc, perc, Dur: 3' a 4', Obs: Escritura proporcional con elementos gráficos, Israel.

[O-102] Arcanos, 1971, perc, Dur: abierta, Obs: Partitura gráfica, Israel.

[O-103] Konstellation II, 1971, orq cdas, Dur: abierta, Obs: Partitura gráfica, Isarel.

[O-104] Trigon, 1971, vn (va), vc, pf, Dur: abierta, Estr: Museum Tel Aviv, Chamber Ensemble, Samuel Lieberson Prize Contest, Tel Aviv, 1972, Ed: Ariadne Musikverlag, Viena (23 de julio de 1974), Obs: Partitura gráfica, Israel.

[O-105-111] Monodrama, 1971, perc, Dur: abierta (7 partes), Obs: Cada una de las siete partes puede ser ejecutada por separado, partitura grafica, Israel.

[O-112] Kolot - Voices, 1971, arp, Dur: abierta, Estr: International Harp Competition (Premio para composición de arpa), Tel Aviv, 1971, Ed: IMI 242 (1 de julio de 1972), Obs: Partitura gráfica, Israel.

[O-113] Canticas, 1971, S, A, CT, T, Bar, B, Dur: abierta, Obs: Partitura gráfica, Israel.

[O-114] Annabel Lee, 1971, cb, Dur: abierta, Obs: Partitura gráfica, Israel.

[O-115] Akiba Ben Yosef, 1972, Mez, S, T, Bar, comx, coinf, narr, orq: 2 fl, 2 ob, 2 cl, 2 fg, 4 cor, 2 tpt, 3 tbn, 1 tu, perc (3 percusionistas), cel, pf, 12 vn I, 12 vn II, 8 va, 8 vc, 4 cb, Dur: 25', Text: Recha Freier, Estr: Testimonium III, Stella Richmond (soprano), Rema Samsonov (soprano) Mira Zakkai (contralto), Menasse Hedjas (tenor), Jerome Barry, Willi Haparnas (barítonos), Coro de niños 
del Ledugma School, Jerusalén, Yitzhak Shilo (narrador), Orquesta Sinfónica de Jerusalén, Juan Pablo Izquierdo (director), Jerusalén Theatre, 26 de febrero de 1974, Ed: IMI 268 (15 de mayo de 1973), Obs: Commissioned by the Israel Composers Fund" (encargo del Fondo del Compositor de Israel), "7-II-1972".

[O-116] Actus, 1972, pf, Dur: 7’.

[O-117] Etude, 1972, orq, Dur: abierta, Ed: Ariadne Musikverlag, Viena (23 de julio de 1974), Obs: Partitura gráfica, Israel.

[O-118] Acting (Actuación), 1972, orq, Dur: abierta, Ed: Ariadne Musikverlag, Viena (23 de julio de 1974), Obs: Partitura gráfica, Israel.

[O-119] Actions for Piano (Acciones para piano), 1972, pf, Dur: abierta, Estr: New Dimensions in Music (Nuevas dimensiones en música), Zmira Lutsky (piano), Tel Aviv Museum, 4 de abril de 1974. Concierto en Homenaje al compositor en sus 75 años, Ingo Schulz (piano), Berlín, 16 de septiembre de 2006. Ed: IMI 268 (15 de mayo de 1973), IMI 7947 (21 de abril de 2011), Obs: Partitura gráfica, Israel.

[O-120] An Anna Blume (Para Anna Blume), 1972, S, A, Bar, Dur: abierta, Text: Kurt Schwitters, Estr: New Dimensions in Music (Nuevas dimensiones en música), Ady Etzion (soprano), Mira Zakai (alto), Jerome Barry (barítono), Barry Silverman (percusión), Tel Aviv Museum, 8 de marzo de 1973, Ed: Ariadne Musikverlag, Viena (23 de julio de 1974), Obs: Partitura gráfica, Israel.

[O-121] Eleven Tombstones (Once lápidas), 1972, A, cor, 2 tpt, tbn, tu, perc (1 percusionista), tape, Dur: 14', Text: Bertolt Brecht, Cesare Pavesa, Dylan Thomas, fuente anónima, Biblia, Estr: Testimonium, III, Wise Auditorium of the Hebrew University, 27 de febrero de 1974, Ed: IMI 295 (1 de enero de 1974), Obs: Escritura proporcional con elementos gráficos, Israel.

[O-122] He engraves Signs into the Heavenly Sphere (Él graba signos en la esfera celestial), 1972, VV, perc, Dur: abierta, Text: Gershom Sholem, Estr: New Dimensions in Music (Nuevas dimensiones en música), Ady Etzion (soprano), Mira Zakai (contralto), Jerome Barry (barítono), Barry Silverman (percusión), Tel Aviv Museum 8 de marzo de 1973; Belcanto Ensemble, Dietburg Spohr (director), Stuttgart 1979, Obs: Partitura gráfica, Israel.

[O-123] Hommage to Picasso (Homenaje a Picasso), 1972, VV, perc, Dur: abierta, Text: Gertrude Stein, Estr: New Dimensions in Music (Nuevas dimensiones en música), Ady Etzion (soprano), Mira Zakai (contralto), Jerome Barry (barítono), Barry Silverman (percusión), Tel Aviv Museum, 8 de marzo de 1973, Obs: Partitura gráfica, Israel.

[O-124] Vera la morte (Vendrá la muerte), 1972, V, perc, Dur: abierta, Text: Cesare Pavese, Estr: Adi Etzion (soprano), Barry Silverman (percusión), Tel Aviv, 14 de enero de 1973, Ed: Ariadne Verlag, Viena, (23 de julio de 1974), Obs: Partitura gráfica, monodrama, Israel.

[O-125] Tetralog, 1972-74 (cada una de las cuatro partes pueden ser ejecutadas por separado).

[O-126] 1: Música para piano y vientos (Premonitions) (Premoniciones), 1972, Dur: abierta, Estr: New Dimensions in Music (Nuevas dimensiones en música), 28 de julio de 1975, Obs: Partitura gráfica, Isarel.

[O-127] 2: Música para piano y cuerdas (Night Music) (Música nocturna), 1972, Dur: abierta, Estr: New Dimensions in Music (Nuevas dimensiones en música), 28 de junio de 1975, Obs: Partitura gráfica. Israel.

[O-128] 3: Música para piano y percusión (Dialogue) (Diálogo) 1974, Dur: abierta, Estr: New Dimensions in Music (Nuevas dimensiones en música), 28 de junio de 1975, Obs: Partitura gráfica , Israel.

[O-129] 4: Música para piano y voz; Dur: abierta, 1974, Obs: Partitura gráfica, Israel.

[O-130] Signals (Señales), 1973, cel, vibr, org, marimbafon, 2 pf, glockenspiel (campanas), Dur: abierta, Estr: New Dimensions in Music (Nuevas dimensiones en música), Tel Aviv Museum, 4 de abril de 1974, Obs: Partitura gráfica, Israel. 
[O-131] In Eius Memoriam (A la memoria de ellos), 1973, orq: 4 fl, 4 ob, 4 cl, 4 fg, 4 tpt, 4 cor, 4 tbn, tu, perc (5 percusionistas), 12 vn I, 12 vn II, 10 va, 10 vc, 8 cb, Dur: 9', Estr: Orquesta Filarmónica de Israel, Zubin Mehta (director), Tel Aviv, 19 de octubre de 1974. Orquesta de Cámara de Chile, Juan Pablo Izquierdo (director), Santiago de Chile. Ed: IMI 302 (1 de julio de 1974), Obs: Subtítulo: "in the day of atonement" (en el día del perdón), "The beauty, O Israel, upon thy high polaces is slain! How are the mighty dallen" ("Tu gloria, Israel, ha perecido en tus montes. ¿Cómo cayeron los poderosos?”), Samuel 2,19, "22-XI-1973”, Israel.

[O-132] Monumentum, 1973, S, A, Bar, narr, orq: 3 fl, 3 ob, 2 cl, 2 fg, 3 tpt, 4 cor, 3 tbn, tu, perc (6 percusionistas), pf, vn I, vn II, va, vc, cb, Dur: 20', Text: Génesis 13,14-15-17, Nekva, Arie Ben Yehuda (el compositor), Estr: Adi Etzion, Mira Zakkai, Jerome Barry, Orquesta de la Radio Kol Israel, Lukas Foss (director), Jerusalén, 4 de junio de 1974, Ed: IMI 283 (24 de julio de 1973), Obs: Escritura proporcional con elementos gráficos, "dedicado al Kibutz Nekva in memoriam Ernst Müller (caído el 8 de diciembre de 1947) y Yasha Ginnesim (caído el 5 de agosto de 1948)", "comisionada por la Israel Broascasting por [el] 25 aniversario del Estado de Israel”, Israel.

[O-133] Ballade for Voice and Irish Minstrel Harp (Balada para voz y arpa irlandesa de ministril), 1973, V, arp céltica, Dur: abierta, Text: fuente Tamil, Estr: Sandra Johanson (arpa), Institute Weizman, Wix Auditorium, Rechovot, 18 de mayo de 1974, Obs: Partitura gráfica, Israel.

[O-134] De profundis (Desde lo profundo), 1973, V, camp tubular, Dur: abierta, Text: Carlos de Rokha, Estr: New Dimensions in Music (Nuevas dimensiones en música), Menashe Hadjes, Tel Aviv Museum, 4 de abril de 1974, Obs: Partitura gráfica, Israel.

[O-135] Momentum (Impulso) 1973, V, pf, org, cel, perc (3 percusionistas), Dur: abierta, Text: anónimo, Estr: New Dimensions in Music (Nuevas dimensiones en música), Tel Aviv Museum, 4 de abril de 1974, Obs: Partitura gráfica, Israel.

[O-136-142] Masada, 1973, (7 partes, cada una puede ser ejecutada por separado), co, orq, Dur: abierta, Obs: Partitura gráfica, Israel.

[O-143] 5 Impromptus pour la Reine Blanche (5 Impromptus para la Reina Blanca) (Andante, Allegretto un poco adagio, Scherzando gracioso, Andante amoroso, Finale), 1974, cl en Si bemol solista, perc (3 percusionistas), Dur: 7', Estr: Giora Feichman (clarinete), Ditbur-Spohr (percusión), Jerusalén, 1974, Obs: Comission from Recha Freier (encargo de Recha Frier), Israel.

[O-144] Ritual, 1974, perc (2 percusionistas), Dur: abierta, Obs: Partitura gráfica, Israel.

[O-145] Introitus, 1974, orq: 4 fl, 4 ob, 4 cl, 4 fg, cfg, 4 tpt, 6 cor, 4 tu, cel, arp, pf, perc (4 percusionistas), 14 vn I, 14 vn II, 10 va, 10 vc, 8 cb, Dur: 5', Obs: Israel.

[O-146] Misa Sine Nomine, 1975-6, 11 partes, rec, org, perc, comx, cada una de las once partes pueden ejecutarse por separado [todas con proyección de diapositivas del gráfico musical], Dur: abierta, Estr: Oster Kirche Bramfeld, Hamburgo, Luz Lansemann (recitante), Anneliese Ubbelohde (órgano), Vocalensamble Hamburg, Kantorei Bramfeld (percusión), Klaus Vetter (director), Hamburgo, 29 de junio de 1980, Ed: IMI 7163 (8 de marzo de 1998), Fon: CD ma 7, LC 0754, musikart Ingo Schulz (www.musik-art.de), grabación de concierto, Berlín, 12 de septiembre de 1998, Obs: Partitura gráfica, "In memoriam Víctor Jara”, Israel.

[O-147] 1: Bereschít bará elohím et haschamáim weét haáretz (En el principio creó Dios los cielos y la tierra, Génesis 1), 1976, 2 comx, perc (4 percusionistas), Dur: abierta, Text: Biblia, Obs: Israel.

[O-148] 2: Kyrie eleison (Señor ten piedad), 1976, gran comx, Dur: abierta, Text: Misa cristiana, Obs: Israel.

[O-149] 3: Lied (Canción), 1976, narr, org, Estr: Concierto en Homenaje al compositor en sus 75 años, Heinz Barthelmeus (narrador), Ingo Schulz (órgano), Berlín, 16 de septiembre de 2006, Dur: abierta, Text: George Grosz, Obs: Israel.

[O-150] 4: Gloria, 1976, gran comx, 4 gongos (1 percusionista), Dur: abierta, Text: Misa cristiana, Obs: Israel.

[O-151] 5: Chile, 1976, $20 \mathrm{VV}$ mixtas, Dur: abierta, Text: compositor, Obs: Israel. 
[O-152] 6: Credo, 1976, narr, gran comx, org, 4 timp bajos (1 percusionista), Dur: abierta, Text: Misa cristiana, Obs: Israel.

[O-153] 7: Benedictus (Bendito sea), 1976, VV mixtas, 4 címbalos colgados (1 percusionista), Dur: 36', Text: Misa cristiana, Obs: Israel.

[O-154] 8: Ich komme (Yo vengo), 1975, 36 VV mixtas, Dur: abierta, Text:Vladimir Mayakovsky, Obs: Israel.

[O-155] 9: Dona nobis pacem (Danos la paz), 1976, gran comx, Dur: abierta, Text: Misa cristiana, Obs: Israel.

[O-156] 10: Babel, 1976, 6 S, 6 A, 4 T, 4 B, Dur: abierta, Text: Biblia, Obs: Israel.

[O-157] 11: Epilog, 1976, narr, pequeño comx, org, 4 perc, Dur: abierta, Text: Biblia, Obs: Israel.

[O-158] Homenaje a Neruda, 1975, comx: 10 S, 10 A, 10 T, 10 B, orq: 4 fl, 4 ob, 4 cl, 4 fg, 4 tpt, 4 cor, 4 tbn, perc (5 percusionistas), 12 vn I, 12 vn II, 10 va, 10 vc, 8 cb, Dur: 16-18', Text: del compositor, Estr: Coro de la NDR, Orquesta Sinfónica de la Norddeutscher Rundfunk, Juan Pablo Izquierdo (director), Hamburgo. Ed: IMI 402 (1 de octubre de 1975), Obs: "14-VI-1975", moto "Canto General, Los libertadores, cap 38, 7-8, Canto General Yo soy, cap. 15, 25", poema dedicado "a mi madre: Llegó la noche... / y no supe que en mi alma, tus párpados se cerraban sobre mi frente”, "14-VI-1975", Israel.

[O-159] Shoa, 1975, narr masc, cl, clb, pf, perc (3 percusionistas), Dur: 7', Text: desconocido, Obs: "8-IV-1975", Israel.

[O-160] Citizen 1230316 (Radiophonic Poem) (poema radiofónico), 1975, Dur: 26', Obs: Prix Italia 1975.

[O-161] Hommage a Schoenberg (Homenaje a Schoenberg) (I: Leicht, zart, II: langsam, III: Sehr langsam, IV Rasch, aber leicht, VI Sehr Langsam), 1975, 2 fl, 2 ob, 2 cl, 2 fg, 2 tpt, 2 cor, 2 tbn, tu, vn I, vn II, va, vc, cb, Dur: 7', Obs: orquestación de "Sechs kleine Klavierstuecke Op. 19", faltando el V, "2427-VII-1975", Israel.

[O-162] DADAYAmasONG, 1975, S, sax, pf, perc, Dur: abierta, Text: de Franz Mehring, Estr: Musik unserer Zeit (Música de nuestro tiempo), Grupo de Cámara Contact-Ensamble, Erhard Karkoschka (director), Stuttgart, 12 de febrero de 1977, Ed: IMI 493 (1 de junio de 1971), Obs: Partitura gráfica, Israel.

[O-163] Invention (Invención), 1976, fl, pf, Dur: 7’, Estr: Michael Melzer (flauta), Boris Berman (piano), Music Spectrum, Tel Aviv Museum, 22 de enero de 1977, Ed: IMI 492 (1 de febrero de 1976), Obs: Israel.

[O-164-170] Golem (7 partes, cada una de las siete partes puede ser ejecutada por separado), 1976 , 7 VV mx: 2 S, Mez, A, T, 2 B, tape, Dur: 15', Text: compositor, Estr: Testimonium IV (Película de Aryeh Mambush y Nina Mayo), 16 de febrero de 1976, Ed: IMI 480 (1 de enero de 1976), Obs: Partitura gráfica, Israel.

[O-171] Aria, 1976, V fem, Dur: abierta, Text: Giuseppe Ungaretti, Estr: Adi Etzion, Sala Bar Shira, Universidad de Tel Aviv, 25 de enero de 1978, Obs: Partitura gráfica, Israel.

[O-172] Chanson, 1976, V fem, tam-tam, Dur: abierta, Text: Paul Éluard, Estr: New Dimensions in Music (Nuevas dimensiones en música), Emilie Berendsen, Tel Aviv 23 de febrero de 1977. Ölberg Kirche, Bärbel Kaiser, Alto Berlín, 11 de mayo de 1990, Ed: IMI 6774 (11 de noviembre de 1996), Obs: Partitura gráfica, Israel.

[O-173] Schatten (Sombra), 1976, 7 vn, 7 vc, 7 cb, 7 camp tubular (+ 7 gongs) (1 percusionista), Dur: abierta, Estr: New Dimensions in Music (Nuevas dimensiones en música), Joan Franka Williams (director), Tel Aviv, 23 de febrero de 1977, Obs: Partitura gráfica, Israel.

[O-174] Trilogy (Trilogía), 1976 - 78, orq, (cada una de las tres partes puede ser ejecutada por separado): [O-175] 1: Prelude to a Drama (Preludio a un drama), 1976, orq: 3 fl, 3 ob, 3 cl, 3 fg, 4 tpt, 4 cor, 3 tbn, tu, perc (5 percusionistas), 12 vn I, 12 vn II, 10 va, 10 vc, 8 cb, Dur: 8', Estr: Jerusalem Symphony Orchestra, Mendi Rodan (director). Orquesta Filarmónica de Israel, Zubin Mehta (director), Tel Aviv, 1981, Ed: IMI 6069 (1 de enero de 1977), Obs: "In memoriam Klaera Plothe", 
motto: "Time held me green and dying / though I sang in my chains like the sea" (El tiempo me mantuvo verde y moribundo / aunque yo cantaba en mis cadenas como el mar) de Hill Dylan Thomas: Last verse (último verso)”, “ 2-X-1976, Tishre 5737, Erev Yom Kippur”, Israel.

[O-176] 2: Lux in tenebris (Luz en las tinieblas), 1977, orq: 3 fl, 3 ob, 3 cl, 3 fg, 4 tpt, 4 cor, 4 tbn, 12 vn I, 12 vn II, 10 va, 10 vc, 8 cb, Dur: 10', Estr: Orquesta Filarmónica de Israel, Aldo Ceccatto (director), Tel Aviv, 1978, Ed: IMI 6123 (16 de enero de 1978), Obs: Motto: An old, mad man still climbing in his ghost (Un anciano loco que todavía trepa en su espíritu), Dylan Thomas, fragmento de I fellowed sleep (Caí dormido), "Tel Aviv- 25-V-1977”, Israel.

[O-177] 3: Canticum, 1978, orq: cel, pf, 2 arp, org, 24 vn, 10 va, 10 vc, 8 cb, Dur: 7', Obs: Motto, "Rage, rage against the dying of the light" (Rabia, rabia contra la muerte de la luz), fragmento de Dylan Thomas Do not go gentle into that good high (No vayas suavemente en esa buena altura), “Tel Aviv-21-IX-77, Tishre 9, 5738 Erev Jom Kippur”, Israel.

[O-178] Images (Imágenes), 1976, orq cdas, Dur: 7, Estr: Holon Chamber Ensamble, Shalom Ronly-Riklis (director), 1978, Ed: IMI 6084 (1 de enero de 1977), Obs: 'Comissioned by the National Council for Culture and Art for the Holon Chamber Orchestra" (encargo del Consejo Nacional de la Cultura y el Arte para la Orquesta de Cámara Holon), Motto: Job 4,16, “25-XII-1976”, Israel.

[O-179] Rising Night after Night (Levantándose noche tras noche) (I Largo desolato, II Andante appassionato, III Adagio sostenuto, IV Alegro molto moderato, V Allegro dolente), 1977, S, T, B, narr, coinf (12 S), comx $(12,12,12,12)$, orq: $3 \mathrm{fl}, 6 \mathrm{ob}, 3 \mathrm{cl}, 4 \mathrm{fg}, 4 \mathrm{tpt}, 4$ cor, 4 tbn, perc (6 percusionistas), pf, cel, $12 \mathrm{vn} \mathrm{I,}$ 12 vn II, 10 va, 10 vc, 8 cb, Dur: 25', Text: Abba Kovner (hebreo), Estr: Competencia Internacional "Hecht” 1977, Obs: cantata, "25-VII-1977", Primer Premio Competencia Hecht, Israel.

[O-180] Annabel Lee, 1977, VV (un narrador y una cantante fem), pf, cel, org, arm, clavicémbalo, perc y tape, Dur: abierta, Text: Edgar Allan Poe, el compositor, Estr: New Dimensions in Music (Nuevas dimensiones en música), Emilie Berendsen (mezzosoprano), The Israel Museum, Jerusalén, 28 de enero de 1978, Obs: Partitura gráfica, Israel.

[O-181] Sound Poem (Poema sonoro), 1977, A, pf, Dur: abierta, Text: Hugo Ball, Estr: New Dimensions in Music (Nuevas dimensiones en música), Emilie Berendsen (mezzosoprano), Zmira Lutzkz (piano), The Israel Museum, Jerusalén, 28 de enero de 1978, Obs: Partitura gráfica, Israel.

[O-182] Aura, 1978, orq cdas: 12 vn I, 12 vn II, 10 va, 10 vc, 8 cb, Dur: 14', Obs: "25-IV-1978, Nisan 18, 5738", Israel.

[O-183] Tel Aviv, 1978-83, orq: 4 fl, 4 ob, 4 cl, 4 fg, 4 tpt, 6 cor, 4 tbn, tu, cel, pf, 2 arp, perc (6 percusionistas), 12 vn I, 12 vn II, 10 va, 10 vc, 10 cb, Dur: 12', Obs: Cita del poeta israelí A. Slonsky, Ed: IMI 6456 (16 de febrero de 1984), Israel.

[O-184] Emanación, 1979, orq: 2 fl, 3 ob, 3 cl, 3 fg, 1 cfg, 4 tpt, 4 cor, 4 tbn, 1 tu, perc (4 percusionistas), 14 vn I, 14 vn II, 12 va, 10 vc, 8 cb, Dur: 7', Obs: "Hamburgo 31-VII-1979", Alemania.

[O-185] Nacht (Noche), 1979, comx a cappella (48 voces), Dur: 8', Text: compositor, Estr: Das neue Werk (La obra nueva), Iglesia St. Nikolai, Coro des NDR, Klaus Vetter (director), Hamburgo, 25 de febrero de 1981, Ed: IMI 7217 (1 de septiembre de 1999), Obs: cita de diarios de "Hamburgo del 21/22 de abril de 1979", "Hamburgo-7-VIII-1979", Alemania.

[O-186] Ayn, 1979, orq: 4 fl, 4 cl, 28 vn, 10 va, 10 vc, 8 cb, cel, arp, pf, perc (8 percusionistas), Dur: 10', Obs: Motto Gerschon Scholem sobre la Kabalah, "Hamburgo 1-XI-1979", Alemania.

[O-187] Palindrom, 1979, (vocalización), cofem, perc, Dur: Abierta, Estr: Apertura de la exposición "Musikalische Graphik von León Schidlowsky, Staatsgalerie, Stuttgart", "Vokal-Ensemble Neue Musik der Stuttgartes Musikhochschule”, Dietburg Spohr (director), Stuttgart, 16 de noviembre de 1979, Obs: Partitura gráfica, Alemania.

[O-188] Klagemauer (Muro de los Lamentos), 1979, rec, co, flexatono, vibr, cl, pf, camp, gongos, glass harmonica, tam-tam y grabadora, Dur: abierta, Text: Biblia, Obs: Partitura gráfica, Alemania. 
[O-189] Tod einer Schauspielerin (La muerte de una actriz), 1979, conj mx de cantantes con máscara, Dur: abierta, Text: collage del compositor, Obs: Partitura gráfica, Alemania.

[O-190-196] Deutschland, ein Wintermärchen (Alemania, un cuento de invierno) (siete partes), 1979, co, narr, sol, pf, perc y conj ca, Dur: abierta, Estr: Concierto en Homenaje al compositor en sus 75 años, Karl-Heinz Barthelmeus (narrador), Rachel Schidlowsky (piano), Bern Vogel-Hjórleifur Jónsson (percusión), Coro Ölberg, Ingo Schulz (director), Berlín, 17 de septiembre de 2006, Fon: CD ma 34, LC 00754, musikart Ingo Schulz (www.musik-art.de), grabación del estreno Obs: Partitura gráfica, el título proviene del libro homónimo de Heinrich Heine, Alemania. Ver estudio de Daniela Fugellie Videla, en RMCh, LXVI/218 (julio-diciembre, 2012), pp. 7-37.

[O-197-203] Der schwarze Gott (El Dios Negro)（siete partes), 1980, V fem, cofem, fl, arp, org, Dur: abierta, Text: Klabund Alfred Henschke, Hamburgo, Estr: Katrin Plümer (flauta), Antje B. Schmidt, Olaf Trenn y Ingo Schulz (percusión), Coro de Mujeres Ölbergkirche, Ingo Schulz (director), Berlín, 23 de septiembre de 2000, Ed: IMI 7164 (8 de marzo de 1998), Fon: CD ma 17, LC 00754, musikart Ingo Schulz (www. musik-art.de), grabación del estreno; Obs: Hamburgo, partitura gráfica, Alemania.

[O-204] Lamento, 1980, orq: 4 fl, 4 ob, 4 cl, 3 fg, 1 cfg, 4 tpt, 4 cor, 3 tbn, 1 tu, perc ( 7 percusionistas), 14 vn I, 14 vn II, 10 va, 10 vc, 8 cb, Dur: 7', Estr: Ralf Peter (contratenor), Ensamble PanArte, Errico Fresis (director), Saarbruecken 28 de marzo de 1999, Obs: "Hamburgo 20-I-1980", Alemania.

[O-205] Cuatro canciones, 1980, S, fl, cl, tbn, perc (3 percusionistas), Dur: 6', Text: poesía precolombina, Ed: IMI 7131 (25 de febrero de 1997), Obs: “4/9-IX-1980”, Alemania.

[O-206] Berlín 80, 1980, orq: 4 fl, 3 ob, 3 cl, 3 fg, 1 cfg, 4 tpt, 6 cor, 3 tbn, 1 tu, perc ( 5 percusionistas), cel, pf, 21 vn, 10 va, 10 vc, 8 cb, Dur: 15’ (7-8), Obs: Berlín, “28-X-1980”, Alemania.

[O-207] Der heilige Sand (La arena sagrada), 1980, co a cappella (7S, 7 A, 7T, 7B), Dur: 7', Text: compositor, Ed: IMI 7632 (22 de diciembre de 2005) o 7218 (1 de septiembre de 1999), Obs: Cita del Archivo de la ciudad de Worms y de Martin Buber, a su vez: "Heiliger Sand / Sand und Erde / Erde und Asche / Heiliger Sand / Nur der Wind / Stau und Sand / Sand und Steine / Heiliger Sand" (Arena sagrada/ Arena y tierra/ Tierra y ceniza/ Arena sagrada/ Solo el viento/ Marea y arena/ Arena y piedra/ Arena sagrada) "texto del compositor, aclaración en alemán como se debe cantar ya que está escrito en forma serial, 19-XI-1980”, Alemania.

[O-208] Quintet, 1980, fl, ob, cl, fg, cor, Dur: abierta, Estr: Exposición de Gráfica Musical, Hochschule für Müsik und Darstellende Kunst, Studio Neue Musik, Kunsthaus, conjunto de vientos de metal de la clase del Profesor Robert Hinze, León Schidlowsky (director), 30 de abril de 1980, Obs: Partitura gráfica, Alemania.

[O-209] Nada, 1980, V fem, Dur: abierta, Text: compositor, Estr: Ulrike Bartsch (voz femenina), Berlín, 10 de septiembre de 2011, Obs: Partitura gráfica, Alemania. Estreno efectuado durante el Concierto conmemorativo para los 80 años del compositor y presentación del libro Musikalische GraphikGraphic Music. Leon Schidlowsky (David Schidlowsky [Hg./Ed.]) WVB Berlin, Berlín 2011.

[O-210] Gilgul, 1980 pf, Dur: abierta, Estr: Konzert-Matinee, exposición, "Leon Schidlowsly. Musikalische Graphik", Carol Morgan (piano), Ludwigshafen, 25 de abril de 1982, Ed: IMI 7934 (28 de julio de 2010), Obs: Partitura gráfica, Alemania.

[O-211] Für Klavier (Para el piano), 1980, pf, Dur: abierta, Estr: Ingo Schulz (piano), Berlín, 29 de marzo de 1992, Ed: IMI 7948 (21 de abril de 2011), Obs: Partitura gráfica, Alemania.

[O-212] If we die (Si morimos) (Aria) 1981, V fem, Dur: abierta, Text: Ethel Rosenberg, Estr: ÖlbergKirche, Bärbel Kaiser (Contralto), Berlín, 11 de mayo de 1990, Obs: Partitura gráfica, Alemania.

[O-213] Adieu, 1982, Mez, pequeña orq: 2 fl, 2 ob, 2 cl, 2 fg, tpt, cor, tbn, arp, perc (1 percusionista), 12 vn, 4 va, 4 vc, 2 cb, Dur: 14', Text: Arthur Penn, Estr: Ensamble New Dimensions, John Frank Williams (director), Jerusalén, 1982, Ed: IMI 6358 (5 de mayo de 1982), Obs: “Tel Aviv 21-II-1982”, Israel.

[O-214] Yizkor (In memoriam), 1982, música incidental, grupo teatral grande, Dur: abierta, Text: compositor, Obs: Comisionada por el director de Teatro Nisan Nativ, Estr: Conferencia y Festival 
Internacional de Teatro Judío, Estudio de Danza y Teatro Nissan Nativ, Jerusalén, 6-7 de julio de 1982, Obs: Partitura gráfica, Israel.

[O-215] Ode, 1982, cofem (10 S, 10 A), tbn, arp, pf-cel (1 percusionista), org, arm, perc (4 percusionistas), orq cdas: 10 vn I, 10 vn II, 7 va, 7 vc, 4 cb, Dur: 20', Text: fuentes litúrgicas, Estr: Testimonium VI, Coro Rinat y miembros de la Orquesta Sinfónica de Jerusalén, Juan Pablo Izquierdo (director), Tel Aviv Museum, 30 de enero de 1983, Ed: IMI 6389 (15 de enero de 1983), Obs: "Comisionado por Testimonium”, "Dedicado a Recha Freier", "28-VII-1982”, Israel.

[O-216] Voices, 1982, comx, Dur: abierta, Estr: Coro de la Ölberggemeinde, Ingo Schulz (director), Berlín 29 de marzo de 1992, Obs: Partitura gráfica, Israel.

[O-217] 1982 - 85 Amerindia (cada una de las cinco partes puede ser tocada por separado:

[O-218] 1: Preludio, 1982, orq: 4 fl, 4 ob, 4 cl, 4 fg, 4 tpt, 6 cor, 3 tbn, tu, perc (4 percusionistas), 12 vn I, 12 vn II, 10 va, 10 vc, 8 cb, Dur: 12', Estr: OSCH, Juan Pablo Izquierdo (director), Santiago de Chile, 1982, Ed: IMI 6377 (15 de enero de 1983), Obs: Moto: "fragmento de un Texto Quechua del siglo XVI traducido por Jesús Lara en el siglo XVII”, "Tel Aviv 14-4-1982”, Israel.

[O-219] 2: Los heraldos negros, 1983, narr, orq: 14 vn I, 14 vn II, 10 va, 10 vc, 8 cb, arp, pf, perc (5 percusionistas), Dur: 7', Text: César Vallejo (Los heraldos negros), Temporada Internacional, Estr: José Soza (narrador), OSCH, Juan Pablo Izquierdo (director), Santiago de Chile, 6 y 7 de agosto de 1999, Ed: IMI 6441 (6 de febrero de 1984), Obs: "Tel Aviv 4-VI-1983”, Israel.

[O-220] 3: Sacsahuamán, 1983, orq: 4 fl, 4 ob, 4 cl, 4 fg, cfg, 4 tpt, 6 cor, 3 tbn, tu, perc (4 percusionistas), $10 \mathrm{vc}, 8 \mathrm{cb}$, Dur: 8', Ed: IMI 6454 (16 de febrero de 1984), Obs: "Hace muchos años que miré tus piedras Sacsahuaman, halcón imperial. Evoco tu imagen en mi cansado corazón” (LS), "on bar 99 the composer used 'La noche del Inca', as musical quotation" (el compositor empleó en el compás 99 "La noche del Inca” como una cita musical), "Tel Aviv 21-VII-1983", Israel.

[O-221] 4: Era el crepúsculo de la iguana, 1985, narr, orq: 4 fl, 3 ob, 4 cl, 3 fg, 4 tpt, 6 cor, 4 tbn, perc (5 percusionistas), arp, pf-cel (1 percusionista), 14 vn I, 14 vn II, 10 va, 10 vc, 8 cfg, Dur: 12', Text: Pablo Neruda (Canto General), Ed: IMI 6567 (2 de diciembre de 2009), Obs: "Tel Aviv 22-X-1995", Israel.

[O-222] 5: Yo vengo a hablar, 1983, narr, orq: 4 fl, 4 ob, 4 cl, 4 fg, 4 tpt, 6 cor, 4 tbn, perc (5 percusionistas), 14 vn I, 14 vn II, 10 va, 10 vc, 8 cb, Dur: 7', Text: Pablo Neruda (Alturas de Macchu Picchu, 7 fragmentos), Estr: Mario Lorca (narrador), OSCH, Rodolfo Fischer (director), Santiago de Chile, 16-17 de abril de 2004, Ed: IMI 6455 (16 de febrero de 1984), Israel.

[O-223] Eclipse, 1983, clb, fl muta, cel, pf, arp, perc (1 percusionista), Dur: 11', Estr: Vigésimo aniversario del Israel Music Institute, Avigail Arnheim (clarinete bajo), 30 de junio de 1987. Goethe Institut, Concierto de Homenaje a Anton Webern y a su discípulo Fré Focke, Francisco Gouet (clarinete bajo), Santiago de Chile, 5 de junio de 1996, Ed: IMI 6492 (15 de diciembre de 1984), Obs: "Tel Aviv 3-IX-1983", Israel.

[O-224] Motu propio, 1983, comx, perc, Dur: abierta, Estr: Coro de la Ölberggemeinde Berlín, Völker Frischling (percusión), Ingo Schulz (director), Berlín, 29 de marzo de 1992, Obs: Partitura gráfica.

[O-225] Toccata, 1983, perc, Dur: abierta, Estr: Concierto en Homenaje al compositor en sus 75 años, Bern Vogel-Hjórleifur Jónsson (percusión), Berlín, 16-17 de septiembre de 2006, Ed: IMI 7928 (04 de enero de 2011), Fon: CD ma34, LC 00754, musikart ingo schulz (www.musik-art.de), grabación del estreno, Obs: Partitura gráfica, Israel.

[O-226] Missa in Nomine Bach (Misa en nombre de Bach) (Benedictus, Agnus Dei, Credo, Hosanna), 1984, comx (4 S, 4 A, 4 T, 4 B), conj ca: fl, ob, cl, clb, vn, va, vc, 8 percusionistas, perc (1 percusionista), Dur: 14', Text: Misa cristiana, Estr: Grupo de Cámara de la Orquesta Filarmónica de Israel, Coro de Cámara Rinat, Shalom Ronly-Riklis (director), Tel Aviv, 1985, Ed: IMI 6482 (5 de diciembre de 1984), Obs: "Tel Aviv, 20-09-1984", Israel.

[O-227] Laude, 1984, comx (5-10 S, 5-10 A, 5-10 T, 5-10 B), org (ad libitum), orq: 2 fl, 2 ob, 2 cl, 2 fg, 2 tpt, 2 cor, 1 tbn, perc (1 percusionista), 6 vn I, 6 vn II, 4 va, 3 vc, 2 cb, Dur: 20', Text: Salmo 130, 
Estr: Kölner Kantorei y Coro de Cámara Kibutz, Shalom Ronly-Riklis (director), Jerusalén 1985, Ed: IMI 6483 (5 de diciembre de 1984), Obs: "on the 300th anniversary of the birth of J.S. Bach" (en los 300 años del nacimiento de Bach), "Tel Aviv Tishrei 16-5745, 12 de octubre de 1984", Israel.

[O-228] Choral, 1984, comx, pf, org, arp, cel, perc, Dur: abierta, Text: Biblia, Estr: Coro de la Ölberggemeinde, Habakuk Traber (órgano), Uli Domay (celesta), Reinhard Hoffmann (clavecín), Ingo Schulz (piano), Volker Frischling (percusión), Berlín, 29 de marzo de 1992, Obs: Partitura gráfica, Israel.

[O-229] Prelude, 1984, comx (vocalización), pf, org, arp, cel, Dur: abierta, Estr: Coro de la Ölberggemeinde, Habakuk Traber (órgano), Uli Domay (celesta), Reinhard Hoffmann (clavecín), Ingo Schulz (piano y dirección), Berlín 29 de marzo de 1992, Obs: Partitura gráfica, Israel.

[O-230] Balada, 1985, vn, orq: 2 fl, 2 ob, 2 cl, 2 fg, cfg, 2 tpt, 4 cor, 2 tbn, tu, perc (4 percusionistas), 12 vn I, 12 vn II, 10 va, 10 vc, 8 cb, Dur: 18', Estr: Jair Kless (violín), Orquesta de la Radio Kol Israel, Shalom Ronly Riklis (director), Tel Aviv 1990, Ed: IMI 6705 (2 de abril de 1992), Obs: "Commisioned by the Tel Aviv Foundation for Literature and Art" (encargo de la Fundación de Tel Aviv para la Literatura y el Arte), "Dedicated to Yair Kless" (Dedicado a Yair Kless), Israel.

[O-231] Bereshit, 1985 coinf (34 voces divididas en el escenario), Dur: 3', Text: Génesis 1,1, Estr: Coro Oranim, C. Barkai (director), Tel Aviv, 1987. Ed: IMI 6566, Obs: "Comissioned by the Art's Council (Ministry of Education)" (Encargo del Consejo para el Arte, Ministerio de Educación), "dedicated to my grandchildren" (dedicado a mis nietos), "21-XII-1985", Israel.

[O-232] Trilogy, 1986 orq (cada una de las tres partes puede ser ejecutada por separado):

[O-233] 1: Cantus, 1986, orq: $2 \mathrm{fl}, 1 \mathrm{fl}$ picc, $1 \mathrm{fl} \mathrm{en} \mathrm{Sol,} 2 \mathrm{ob}, 1 \mathrm{ci}, 2 \mathrm{cl}, 1 \mathrm{cl}$ picc, $1 \mathrm{clb}, 2 \mathrm{fg}, 1 \mathrm{cfg}$, 4 tpt, 6 cor, 3 tbn, 1 tu, perc (4 percusionistas), 14 vn I, 14 vn II, 10 va, 10 vc, 8 cb, Dur: 14', Ed: IMI 6593 (13 de marzo de 1995), Obs: "21-II-1986”, Israel.

[O-234] 2: Exequiae (Exequias), 1986, comx (14 S, 14 A, 14 T, 14 B), orq: 4 fl, 4 ob, 4 cl, 4 fg, tpt, 4 cor, 4 tbn, perc (6 percusionistas), Dur: 14', Text: compositor, Requiem, Ed: IMI 6594. Obs: "Tel Aviv 17-VII-1996", Israel.

[O-235] 3: Hymn to the Dawn (Himno a la aurora), 1986-7, orq: 4 fl, 4 ob, 4 cl, 4 fg, 6 cor, 4 tpt, 4 tbn, perc (4 percusionistas), cel, pf, arp, 14 vn I, 14 vn II, 10 va, 8 cb, Dur: 12', Ed: IMI 6602, Obs: Motto: "Mattina: M'illumino / d'inmenso (Giuseppe Ungaretti), "Dawn: I illuminate myself / through inmensity (Giuseppe Ungaretti) (Aurora: me ilumino/ a través de la inmensidad), "Tel Aviv 28-II-1987", Israel.

[O-236] A Man between Stones (Un hombre entre piedras), 1987, 4 piedras (+ percusión) (1 percusionista), Dur: abierta, Obs: Partitura gráfica, Israel.

[O-237] Missa Dona Nobis Pacem (Misa Dános la paz) (Introitus, Kyrie, Graduale, Credo, Benedictus, Sequentia, Responsorium, Agnus Dei), 1987, comx a cappella, Dur: 30', Text: Misa cristiana (latin), Ed: IMI 6699, Obs: "24-VIII-1979 Tel Aviv - Israel".

[O-238] Elegy (Elegía), 1988, orq: 4 fl, 4 ob, 4 cl, 4 fg, 4 tpt, 6 cor, 4 tbn, tu, cel, arp, pf, perc (4 percusionistas), 14 vn I, 14 vn II, 10 va, 10 vc, 8 cb, Dur. 12', Ed: IMI 6758, Obs: Israel.

[O-239] Laudatio (Alabanza), 1988, orq: 4 fl, 4 ob, 4 cl, 4 fg, 1 cfg, 4 tpt, 6 cor, 4 tbn, perc (4 percusionistas), 14 vn I, 14 vn II, 10 vc, 8 cb, Dur: 10', Ed: IMI 6759, Obs: "on the 40th Anniversary of the State of Israel, to my sons Yuval and Noam" (en el cuadragésimo aniversario del Estado de Israel, a mis hijos Yuval y Noam), "Tel Aviv 22-9-1988”, Israel.

[O-240] Piano Quartet (Cuarteto con piano), 1988, pf, vn, va, vc, Dur: 7', Estr: Tel Aviv Piano Quartet, Tel Aviv, 27 de enero de 1990, Estr Instituto Goethe, Ensemble Contemporáneo de la Facultad de Artes de la Universidad de Chile, Aliocha Solovera (director), Santiago de Chile, 14 de octubre de 2002, Ed: IMI 6755, Fon: Ensamble Contemporáneo. CD. Santiago de Chile, Universidad de Chile, 2003, Obs: “Tel Aviv 28-II-1988”, Israel. Reseña del fonograma por Julia Grandela del Río en RMCh, LVIII/201 (enero-junio, 2004), pp. 120-121. 
[O-241] Cuarteto de cuerdas, 1989, vn I, vn II, va, vc, Dur: 12', Ed: IMI 6788 (14 de julio de 1996), Obs: "Tel Aviv 31-1-1989", Israel.

[O-242] An den Knaben Elis (Al niño Elis), 1989, 16 sol (4 S, 4 A, 4 T, 4 B) y perc (3 percusionistas), Dur: 12', Text: Georg Trakl, Ed: IMI 6819, Obs: "fuer Nils-Christian Kaehler, geb. 5-5-1963 gest. 12-6-1985" (para Nils-Christian Kaehler, nació el 5 de mayo de 1963; falleció el 12 de junio de 1985), “Tel Aviv 14-VII-1989”, Israel.

[O-243] Caleidoscope (Caleidoscopio), 1989, orq: 4 fl, 4 ob, 2 cl, 4 fg, 6 cor, 4 tpt, 4 tbn, perc (4 percusionistas), vn I, vn II, va, vc, cb, Dur: 7', Ed: IMI 6826, Obs: “Tel Aviv 23-X-1989”, Israel.

[O-244] Five Pieces for Harp (Cinco piezas para arpa), 1989, arp, Dur: 10', Estr: Festival Internacional de Arpa, Jerusalén, 1991, Ed: IMI 6822 (07 de abril de 1992), Obs: "28-XII-1989", Israel.

[O-245] Prelude (Preludio), 1990, orq: 4 fl, 4 ob, 4 cl, 4 fg, cfg, 4 cor, 4 tpt, 4 tbn, tu, perc (3 percusionistas), 14 vn I, 14 vn II, 10 va, 10 vc, 8 cb, Dur: 12', Ed: IMI 6825, Obs: "21-II-1990", Israel.

[O-246] Clamavi (Clamaba), 1990, vn, orq ca: fl, cl, tpt, cor, pf, perc (1 percusionista), vn I, vn II, va, vc, cb, Dur: 21', Obs: Israel.

[O-247] Shadows (Sombras), 1990, conj ca: fl, cl, arp, pf, perc (1 percusionista) 2 vn, va, vc, cb, Dur. 12', Estr: Ensemble Buchmann-Metha School of Music, Ethan Schmeisser (director), Tel Aviv, 2006, Ed: IMI 6852, Obs: Israel.

[O-248] In memoriam Luigi Nono, 1990, va, vc, cb, Dur: 14', Estr: Trio Bass (grabación 1990). Trio Tel Aviv, Tel Aviv, 7 de enero de 1991, Ed: IMI 6839, Obs: "Trio Bass gewidmet" (dedicada al Trío Bass), Obs: Israel.

[O-249] Trilogy (Trilogía) (Prelude, Cantico, Allegro furioso), 1990, 2 pf, Dur: 7', Ed: IMI 6867. Obs: "Dedicated to Prof. Gisela and Erich Andreas" (Dedicada a los profesores Gisela y Erich Andreas), “Tel Aviv 14-IX-1990”, Israel.

[O-250] Toccata, 1991, pf, Dur: 8', Estr: Unerhörte Musik (Música inaudita), Auditorium B. K.A., Elizabetha Sternlicht (piano), Berlín, 21 de septiembre de 1993, Ed: IMI 7006 (28 de noviembre de 1994), Obs: "Tel Aviv 22-X-1990”, Israel.

[O-251] Arabesque, 1991, fl traversa, Dur: 8', Ed: IMI 6912 (8 de julio de 2002), Estr: Noam Lior (flauta), Boston University, 11 de abril de 1993. Concierto en Homenaje al compositor en sus 75 años, Andrea Welte (flauta), Berlín, 16 de septiembre de 2006, Obs: "For Noam Lior" (Para Noam Lior), Israel.

[O-252] Septimino, 1991, fl, ob, cl, fg, tpt, cor, tbn, Dur: 12', Estr: Música Nova, solistas bajo la dirección de Arthur Weisberg, Museo Tel Aviv, Tel Aviv, 6 de marzo de 1999. Orquesta de Cámara de Chile, Juan Pablo Izquierdo (director), Teatro Municipal de Nuñoa, Santiago de Chile, 21 de junio de 2013, Ed: IMI 6911, Obs: "Tel Aviv 4-X-1991”, "In memoriam Edgar Varèse”, Israel.

[O-253] Todesfuge (Fuga de la muerte), 1991, cofem (2 S, $4 \mathrm{Mez}, 2 \mathrm{~A})$, perc (instrumentos de percusión interpretados por las cantantes), Dur: 15', Text: Paul Celan, Ed: IMI 6913 (23 de julio de 1998), Obs: 28 de agosto de 1991, "For The Bel Canto Ensemble" (Para el Ensemble Bel Canto), Israel.

[O-254] Polyphony VII (Polifonía VII), 1991, orq: 4 fl, 4 ob, 4 cl, 5 fg, 4 cor, 4 tpt, 4 tbn, tu, perc (5 percusionistas), cel, arp, pf, org, 14 vn I, 14 vn II, 10 va, 10 vc, 8 cb, Dur: 14', Ed: IMI 6908, Obs: "Tel Aviv 7-VIII-1991", Israel.

[O-255] Exhortatio, 1991, orq: 4 fl, 3 ob, 4 cl, 3 fg, 4 tpt, 6 cor, 4 tbn, perc ( 7 percusionistas), 14 vn I, 14 vn II, 10 va, 10 vc, 8 cb, Dur: 12', Ed: IMI 6914, Obs: "In memoriam Lev Davidovich Bronstein, murdered on the 20th August 1940" (In memoriam Lev Davidovich Bronstein, asesinado el 20 de agosto de 1940), "Tel Aviv-12-XI-1991”, Israel.

[O-256] Carrera, 1991, narr, orq: 4 fl, 2 ob, 2 cl, 2 fg, 4 tpt, 3 cor, 3 tbn, 1 tu, perc (7 percusionistas), vn I, vn II, va, vc, cb, Dur: 14', Text: Pablo Neruda, Estr: Egbert Junghaus (narrador), Rundfunk 
Sinfonie Orchester Berlin, Juan Pablo Izquierdo (director), Berlín, 3 de diciembre de 1993, Ed: IMI 6861, Obs: Israel.

[O-257] Salmos, 1992, narr, comx, orq: 2 fl, 2 ob, 2 cl, 2 fg, 3 cor, 3 tpt, perc (4 percusionistas), arp, cel, org, vns, vas, vc, cb, Dur: 14', Text: Biblia (traducción al español), Ed: IMI 6938, Obs: Israel.

[O-258] Morgengebet (Oración de la mañana), 1993, cl, Dur: 7', Obs: "Giora Feidman gewidmet" (Dedicada a Giora Feidman) "Berlín 21-Adar-5753" "14-Maerz 1993”, Obs: Alemania.

[O-259] Der Dibbuk, ópera en 3 actos, 1993, S, 4 A, 5 Bar, 12 T, 7 B, comx: 5 S, 5 A, 5 T, 5 B, comx: 5 T, 5 B, coinf: 14 voces, cofem: 5 S, 5 A, 4 fl, $1 \mathrm{cfg}, 4$ tpt, 4 cor, 4 tbn, 1 tu, arp, cel, pf, arm, mand, gui, 7 perc: glockenspiel, xil, vibr, camp tu, crótalos, sierra musical, flexaton, 4 timbles, 4 bongos, 3 tamb pequeños, Rührtrommeln, 5 tom-toms, tamborino, 3 congas, 3 tamb grandes, martillo, eolífono, dontrolado, 3 cajas chinas, guiro, 4 gongs, 4 tam-tams, mar, camp de cristal, 14 vn I, 14 vn II, 10 va, 10 vc, 8 cb, 2 pequeños conjuntos de vientos, Dur: 120', Text: compositor, según Salomon An-Ski, Obs: "gewidmet, all denjenigen, die nie mehr jene Welt erleben können" (dedicado a todos aquellos que nunca más podrán percibir aquel mundo), Berlín, 11 de enero de 1993, 18-Tevet-5753, Alemania.

[O-260] Sealed Room (Pieza sellada), 1993, pequeña orq: fl, ob, cl, fg, cor, tpt, tbn, 2 vn, va, vc, cb, Dur: 8', Estr: Auditorium Mishkenot Haanaruin, solistas de la Orquesta Sinfónica de Jerusalén, Zsolt Nagy (director), Jerusalén, 20 de marzo de 1994, Ed: IMI 6904, Obs: Israel.

[O-261] I will lay Mine Hand upon my Mouth (Yo pondré mi mano sobre mi boca), 1994, orq: 4 fl, 4 ob, 4 cl, 4 fg, 4 tpt, 4 cor, 4 tbn, perc (7 percusionistas), 14 vn I, 14 vn II, 10 va, 10 vc, 8 cb, Dur: 14', Ed: IMI 7004 (28 de noviembre de 1994), Obs: Moto en alemán: Job. 40,4: "Ich lege meine Hand auf meinem Mund" (yo pondré mi mano sobre mi boca), “Tel Aviv 14-IV-1994”, Israel.

[O-262] Am Grab Kafka's (En la tumba de Kafka), 1994, V fem (+ crótalos), proyección de diapositivas, Dur: abierta, Text: Franz Kafka, Estr: Wirsberg-Gymnasiums Wuerzburg, Jutta Meierott (directora), 24 de enero de 1997, Ed: IMI 7009 (28 de noviembre de 1994), Obs: Partitura gráfica, Israel.

[O-263] Silvestre Revueltas, oratorio 1994, narr, orq ca: fl, cl, fg, perc (2 percusionistas), pf, vn, vc, cb, Dur: 14', Text: Pablo Neruda, Estr: Buchmann-Mehta School of Music, Universidad de Tel Aviv, León Schidlowsky (narrador), Elisabeth Wentland (flauta), Tomer Sharoni (clarinete), Doron Laznav (fagot), Shaden Nahra (violín), Omer Ein-Habar (violonchelo), Shira Davidson (contrabajo), Lior Moskovitz (piano), Eran Margalit (percusión), Nadav Ovadia (percusión), Ezequiel Silberstein (director), Tel Aviv, Israel, 16 de junio de 2015.

[O-264] Laudate (Alabad), 1995, comx (12 S, 12 A, 12 T, 12 B), Dur: 14', Text: Salmos (latín, alemán, hebreo), Estr: NDR-Chor, Robin Gritton (director), Hamburgo, 25 de enero de 1996, Obs: Comisionada por la Radiodifusión Nord Deutsche Rundfunk para el "50avo Aniversario de la creación del Coro de la NDR”, Hamburgo, Alemania, Ed: IMI 7024 (16 de junio de 1997), Israel.

[O-265] Absalom, 1995, gran orq: 4 fl, 4 ob, 4 cl, 4 fg, 4 cor, 4 tpt, 3 tbn, 1 tu, 1 cel, 1 arp, perc (5 percusionistas), 14 vn I, 14 vn II, 10 va, 10 vc, 8 cb, Dur: 17’, Ed: IMI 7089 (24 de octubre de 1996), Estr: Primer Premio, Conmemoración de los 65 años de la creación de la Orquesta Filarmónica de Israel, Orquesta Filarmónica de Israel, Zubin Mehta (director), Tel Aviv, julio 1997, Obs: "7VIII-1995", Israel.

[O-266] In memoriam, 1996, vc, pf, perc, Dur: 7', Estr: Alejandro Tagle (violonchelo) y Gonzalo Muga (piano), VI Festival de Música Contemporánea, Santiago, Chile, 1996, Obs: Israel.

[O-267] Threnos (Trío), 1996, fl, va, perc, Dur: 10', Ed: IMI 7099 (26 de diciembre de 1996), Estr: Ensemble Erew Leila, Trieste, Italia, 14 de septiembre de 2008, Obs: "In memoriam Y. Rabin", Israel.

[O-268] The Darkest Night (La noche más oscura), 1997, orq cdas: vn I, vn II, va, vc, cb, Dur: 7', Ed: IMI 7110 (25 de febrero de 1997), Obs: Motto Isaías 7,3-4, "In memoriam the 73 victims" (A la memoria de las 73 víctimas), "7-II-1997”, Israel. 
[O-269] De profundis (Desde lo profundo), 1997, orq cdas, 14 vn I, 14 vn II, 10 va, 10 vc, 8 cb, Dur: 14', Ed: IMI 7111 (25 de febrero de 1997), Obs: “28/1/1997”, Israel.

[O-270-80] Greise sind die Sterne geworden - eine moderne Passion (Oscuras se han transformado las estrellas - una pasión moderna), 1997 (once partes), S, A, Bar, narr, comx, pf, org, arp, cel, perc (3 percusionistas), Dur: abierta ca 60, Text: Heinrich Heine, Georg Trakl, Else Lasker-Schüler, Mascha Kaléko, Erich Fried, M. Gaztrum, Novalis, Biblia), Estr: Antje B. Schmidt (soprano), Britta Süberkrüb (contralto), Michael Hoffmann (barítono), Olaf Trtenn (recitante), Gudrun Schlag (piano), Katrin Plümer (celesta), Antje B. Schmidt (clavecín), Habakuk Traber (órgano), Nicole Hartig-Mathias Lochmann-Thomas Rönnefarth (percusión), 2 coros aumentados del Coro Ölberg, Ingo Schulz (director), Berlín, 25-26 de marzo de 2000, Ed: IMI 7178 (4 de septiembre de 1998), Fon CD ma 13, LC 00754, musikart Ingo Schulz (www.musik-art.de), grabación del estreno, Obs: Partitura gráfica, Israel.

[O-281] Premonitions (Premoniciones), 1997, orq: 4 fl, 4 ob, 4 cl, 4 fg, 4 tpt, 4 cor, 4 tbn, 14 vn I, 14 vn II, 10 va, $10 \mathrm{vc,} 8 \mathrm{cb}$, Obs: "21-XI-1997", Israel.

[O-282] Before Breakfast (Antes del desayuno), (ópera en un acto), 1998, 1 S, orq: 2 fl, 2 ob, 1 ci, 2 cl, 1 clb, 3 fg, 1 cfg, 4 tpt, 4 cor, 3 tbn, tu, 4 timp, xil, gloc, vibr, camp, etc, 5 perc, 12 vn I, 12 vn II, 10 va, 8 vc, 6 cb, Dur: 45', Text: del compositor, según Eugene O’Neill, Ed: IMI 7170 (15 de mayo de 1998), Obs: "Hans Jochen Genzel gewidmet, Berlin" (Dedicada a Hans Jochen Genzel, Berlín), "8-3-1988", Alemania, partitura original extraviada.

[O-283] Seven Nocturnes (Siete nocturnos), 1998, vn, pf, Dur: 7', EstrSemadar Schidlowsky (violín), Rachel Schidlowsky (piano), Berlín, 28 de febrero de 1999, Ed: IMI 7162 (8 de marzo de 1998), Obs: "To Semadar and Rachel” (A Semadar y Rachel), Alemania.

[O-284] Lamento, 1998, CT, conj ca: vn I, vn II, va, vc, cb, arp, perc (1 percusionista), Dur: 7', Text: Heinrich Heine (Romancero), Ed: IMI 7172 (21 de junio de 1998), Estr: Ralf Peter (contratenor), Ensemble Pan Arte, Errico Fresis (director), Saarbrücken, Alemania, 28 de marzo de 1999, Obs: Alemania.

[O-285] Si muero, 1998, narr, fl, arp, org, Dur: 10', Text: Federico García Lorca ("Despedida”, Memento"), Estr: Antje B. Schmidt (recitante femenina), Katrin Plümer (flauta), Tatjana Schütz (arpa), Gunter Kennel (órgano), Ingo Schulz (director), Berlín, 23 de septiembre de 2000, Ed: IMI 7220 (1 de septiembre de 1999), Obs: Alemania.

[O-286] Altazor. In memoriam Vicente Huidobro, 1999, orq: 4 fl, 4 ob, 4 cl, 4 fg, cfg, 4 tpt, 4 cor, 4 tbn, tu, perc (5 percusionistas), arp, cel, pf, 12-14 vn I, 12-14 vn II, 8-10 va, 8-10 vc, 6-8 cb, Dur: 11', Obs: "Berlin 15-I-1999", Alemania.

[O-287] Mahnmal - Memorial, 1999, conj ca: $2 \mathrm{fl}$ picc, 2 ob, 2 cl, 4 fg, 2 tpt, 4 cor, 4 tbn, perc (4 percusionistas), 12-14 vn I, 12-14 vn II, 8-10 va, 8-10 vc, 6-8 cb, Dur: 14', Ed: IMI 7219 (1 de septiembre de 1999), Obs: Subtítulo: "In Erinnerung an die Befreiung von Auschwitz Durch die Rote Armee [27-I-1945]" (En recuerdo de la liberación de Auschwitz por el Ejército Rojo [27-I-1945]), "Berlin 27-I-1999", Alemania.

[O-288] The Sacrifice of Isaac I (Isaaks Opferung) (El sacrificio de Isaac I), 1999, Bar, S, T, B, comx (14 S, $14 \mathrm{~A}, 14 \mathrm{~T}, 14 \mathrm{~B}$ ), orq: $4 \mathrm{fl}, 4 \mathrm{ob}, 4 \mathrm{cl}, 4 \mathrm{fg}, 1 \mathrm{cfg}, 4 \mathrm{tpt}, 4 \mathrm{cor}, 3 \mathrm{tbn}, 1 \mathrm{tu}$, perc (5 percusionistas), 14 vn I, 14 vn II, 10 va, 10 vc, 8 cb, Dur: 21', Text: Biblia, Génesis 22,1-18 [Hebreo]), Ed: IMI 7221 (1 de septiembre de 1999), Obs: "Meine Frau gewidmet" (Dedicado a mi esposa), "2-5-1999", Alemania.

[O-289] El muro, 1999, fl picc, fg, cfg, tpt, cor, tbn, tu, perc (3 percusionistas), Dur: 10', Ed: IMI 7240 (3 de febrero de 2000), Obs: "In memoriam de los caidos en Chile entre 1973-1988", "Berlin, 11-V-1999", Alemania.

[O-290] The Sacrifice of Isaac II (Isaaks Opferung) (El sacrificio de Isaac II), 1999, narr, T, B, Bar, comx, orq: $4 \mathrm{fl}, 4 \mathrm{ob}, 4 \mathrm{cl}, 4 \mathrm{fg}, 4 \mathrm{tpt}, 4 \mathrm{cor}, 3 \mathrm{tbn}, 1 \mathrm{tu}$, perc ( 5 percusionistas), $14 \mathrm{vn} \mathrm{I,} 14 \mathrm{vn}$ II, $10 \mathrm{va}, 10$ vc, 8 cb, Dur: 25', Text: Biblia, Génesis 22,1-18 (traducción al alemán), Obs: "Berlin 28-VI-1999", Alemania. 
[O-291] Meditaciones, 2000, fl, ob, clb, vn, vc, cb, Dur: 11', Estr: Noam Buchman (flauta), Omri Rave (oboe), Sigal Ejtelinger (clarinete bajo), Ela Lutzki (violín), Irit Asias (violonchelo), Sergei Gerlnik (contrabajo), Cristian Eggen (director), Museo Tel Aviv, Tel Aviv, 19 de enero de 2002. Corporación Cultural de Las Condes, XIV Temporada de Conciertos, Parroquia de Nuestra Señora del Rosario, Orquesta de Cámara de Chile, Juan Pablo Izquierdo (director), Santiago de Chile, 14 de mayo de 2008. Teatro Universidad de Concepción, Orquesta Sinfónica de la Universidad de Concepción, Juan Pablo Izquierdo (director), Concepción, Chile, 28 de junio de 2008, Ed: IMI 7241 (3 de febrero de 2000), Obs: Berlín "In memoriam Susanne Schidlowsky", "25-I-2000", Alemania.

[O-292] Three Dialogues (Tres diálogos), 2000, 2 vn, Dur: 7-10', Estr: Yair Kless y Eyal Kless, Jerusalén, 8 de enero de 2001, Ed: 7276 (30 de octubre de 2000), Obs: "Para Yair e Ilan Kless", "A la memoria de Susanne Schidlowsky", Motto: Miguel Hernández: "Las tres heridas" (del Cancionero y Romancero de ausencia), "10-II-2000”, Berlín, Alemania.

[O-293] Evocación, 2000, conj ca: 1 fl, 2 ob, 2 fg, 2 cor, 5 vn I, 5 vn II, 3 va, 3 vc, 1 cb, Dur: 11', Estr: Corporación Cultural de Las Condes, Orquesta de Cámara de Chile, Fernando Rosas (director), Parroquia de San Vicente Ferrer, Santiago de Chile, 8 de mayo de 2001, Teatro Oriente, Santiago de Chile, 9 de mayo de 2001, Teatro Municipal de Nuñoa, Santiago de Chile, 10 de mayo de 2001,, Ed: IMI 7297 (27 de agosto de 2001), Obs: "Evocación está escrita en recuerdo a mi esposa Susanne Schidlowsky (Q.E.P.D.)”, "Berlin, 5-VIII-2000”, Alemania.

[O-294] Tenebrae (Tinieblas), 2000, conj ca: fl picc, fl, fg, cfg, 3 tbn, perc (5 percusionistas), vn I, vn II, va, vc, cb, Dur: 11', Ed: IMI 7277 (30 de octubre de 2000), Obs: "Berlin, 29-VI-2000”, Alemania.

[O-295] Dedalus Death (La muerte de Dédalo), 2000, gran orq: 3 fl, 3 ob, 3 cl, 3 fg, 3 tpt, 4 cor, 3 tbn, 1 tu, perc (3 percusionistas), vibr, pf-cel, 14 vn I, 14 vn II, 10 va, 10 vc, 10 cb, Dur: 14', Ed: IMI 7821 (14 de enero de 2001), Obs: "Berlin 15-VIII-2000", Alemania.

[O-296] Dedalus (Dédalo), 2000, gran orq: picc, 2 fl, 2 ob, 1 ci, 2 cl, 1 clb, 2 fg, 1 cfg, 3 tpt, 4 cor, 1 tu, perc (3 percusionistas) pf-cel, arp, 16 vn I, 14 vn II, 12 va, 10 vc, 8 cb, Dur: 12', Ed: IMI 7282 (19 de marzo de 2001), Obs: "14-IX-2000", Alemania.

[O-297] Vox clamantis in deserto (La voz que clama en el desierto), 2000, 3 fl, 3 ob, 3 cl, 3 fg, 4 tpt, 3 cor, 3 tbn, 1 tu, perc (3 percusionistas), arp, pf, cel, cdas, Dur: 10', Estr: Orquesta Filarmónica de Israel, Mendi Rodan (director), Henry Crown Hall, Jerusalén, 8 de octubre de 2002. Teatro Universidad de Chile, OSCH, Juan Pablo Izquierdo (director), Santiago de Chile, 13 y 14 de mayo de 2011, Ed: IMI 7280 (14 de enero de 2001), Obs: Israel.

[O-298] Dúo, 2001, fl, arp, Dur: 7’, Ed: IMI 7283 (19 de marzo de 2001), Obs: "Katrin Pluemer und Tayjana Schuetz gewidmet” (Dedicado a Katrin Pluemer y Tayjana Schuetz), Israel.

[O-299] Bakasha (Pedido), 2001, V, fl, ob, arp, Dur: 8', Text: Karmi, Estr: Teatrino di San Giovanni, Ensemble Erew Leila, Davide Casali (director), Trieste, Italia, 14 de septiembre de 2008, Ed: IMI 7285 (19 de marzo de 2001), Obs: “Tel Aviv 31-I-2001”, Israel.

[O-300] Christus im Holocaust (Cristo en el Holocausto), 2001, org, Dur: 11', Estr: Guenter Kennel (órgano), Kirche Zum Heiligen Kreuz, Berlín, 29 de marzo de 2002, Ed: IMI 7284 (19 de marzo de 2001), Obs: "Dedicated to Guenter Kennel" (Dedicado a Guenter Kennel), Christus im Holocaust has been written by the work of the Jewish sculptor Ismond Rosen" ( Cristo en el Holocausto está inscrito en la obra del escultor judío Ismond Rosen), "Tel Aviv 11-2-2001", Israel.

[O-301] In memoriam Jorge Peña, 2001, narr, orq: 3 fl, 3 ob, 3 cl, 3 fg, 3 cor, 3 tpt, 3 tbn, perc (3 percusionistas), 10 vn I, 10 vn II, 8 va, 8 vc, 6 cb, Dur: 10', Text: compositor, Estr: Catedral de La Serena, Orquesta Sinfónica Nacional Juvenil, Fernando Rosas (director), La Serena, Chile, 16 de octubre de 2003. Teatro Municipal de Nuñoa, Orquesta Sinfónica Nacional Juvenil, Fernando Rosas (director), Santiago de Chile, 24 de octubre de 2003, Fon: Música para Jorge Peña Hen, publicado por la FOJI (Fundación de Orquestas Juveniles e Infantiles), Orquesta Sinfónica Nacional Juvenil, dirección José Luis Domínguez (director), Santiago de Chile, 2007; Obs: "En memoria de Jorge Peña (asesinado el 16 de octubre de 1973 en La Serena, Chile)”, “Tel Aviv 12-III-2001”, Israel. 
[O-302] Spectra (Espectros), 2002, orq: picc, 2 fl, 2 ob, 1 ci, 2 fg, 4 tpt, 4 cor, 3 tbn, 1 tu, cel, pf, arp, perc (5 percusionistas), $14 \mathrm{vn} \mathrm{I,} 14 \mathrm{vn} \mathrm{II,} 10 \mathrm{va}, 10 \mathrm{vc}, 8 \mathrm{cb}, E d$ : IMI 7527 (26 de mayo de 2003), Obs: "Tel Aviv 1-I-2002", Israel.

[O-303] Transparencias, 2002, fl en Sol, ci, clb, arp, vn, vc, cb, Dur: 11', Ed: IMI 7454 (8 de julio de 2002), Estr: Ensemble Erew Laila, Trieste, Italia 2008, Obs: “Tel Aviv 21-II-2002”, Israel.

[O-304] Adagio, 2002, pequeña orq cdas: vn I, vn II, va, vc, cb, Dur: 10', Estr: Auditorio Sharet, Orquesta de Cámara de Ashdod, Chen Zimbalista (director), Petach Tikva, Israel, 30 de septiembre de 2002, Ed: IMI 7453 (8 de julio de 2002), Obs: “Tel Aviv 27-II-2002”, Israel.

[O-305] Partita, 2002, ob, Dur: 7’, Ed: IMI 7452 (8 de julio de 2002), Obs: "Tel Aviv 11-IV-2002", Israel.

[O-306] Prayer (Oración), 2002, vn, Dur: 7', Estr: The Henry Crown Symphony Hall, Jair Kless (violín), Jerusalén, 3 de noviembre de 2003. Concierto en Homenaje al compositor en sus 75 años, Semadar Schidlowsky (violín), Berlín, 16 de septiembre de 2006, Ed: IMI 7451 (8 de julio de 2002), Obs: “Tel Aviv 9-III-2002". Israel.

[O-307] Serenata, 2002, mand, Dur: 7', Estr: Avi Avital, Italia, 2002, Ed: IMI 7448 (7 de mayo de 2012), Obs: "Tel Aviv 31-V-2002", Israel.

[O-308] Trío “The Swan" (Trío "El cisne”), 2002, vn, va, vc, Dur: 12', Estr: The Henry Crown Symphony Hall, Jair Klees (violín), Gad Lewertoff (viola), Emanuel Gruber (violonchelo), Jerusalén, 3 de noviembre de 2003, Ed: IMI 7524 (26 de mayo de 2003), Obs: "Tel Aviv 18-XI-2002", Israel.

[O-309] And Death shall have no Dominion (Y la muerte no tendrá dominio), 2002, gran orq: 4 fl, 4 ob, 4 fg, 4 tpt, 4 cor, 4 tbn, arp, vn I, vn, va, vc, cb, Dur: 14', Ed: IMI 7526 (26 de mayo de 2003), Obs: Israel.

[O-310] The Secret Garden (El jardín secreto), 2003, fl, ob, cl, clb, arp, Dur: 7', Ed: IMI 7525 (26 de mayo de 2003), Obs: "Tel Aviv 16-XII-2002", Israel.

[O-311] The Song of Sorrow (El cantar de los dolores), 2003, mez, cor, arp, vc, Dur: 10', Text: Giuseppe Ungaretti: "Santo", "Un'altra note" (Otra nota), "Mattina" (Mañana), Estr: "A tribute to León Schidlowsky on his 75th birthday" (tributo a León Schidlowsky en sus 75 años), Hadas Gur (mezzosoprano), Michael Horn, Horn (corno), Dan Weinstein (violonchelo), Gitit Boazson (arpa), Guy Feder (director), Tel Aviv, 2006, Ed: IMI 7523 (26 de mayo de 2003), Israel.

[O-312] Coming Back to Life (Volviendo a la vida), Tango, 2003, vn, pf, Dur: 8', Estr. Emauel Krasowsky (violín), Verse Vaidman (piano), Falsterbo, Suecia, 23 de julio de 2004. Concierto en Homenaje al compositor en sus 75 años, Semadar Schidlowsky (violín), Rachel Schidlowsky (piano), Berlín, 16 de septiembre de 2006, Ed: IMI 7545 (16 de junio de 2004), Obs: "Dedicated to Raquel and Marcelo Kosciak" (Dedicado a Raquel y Marcelo Kosciak), Tel Aviv, 26 de abril de 2003, Israel.

[O-313] Four Songs (Cuatro canciones), 2003, Mez, cl, fg, cor, arp, vn, vc, Dur: 8', Text: Dylan Thomas, Paul Celan, Paul Eluard, Pablo Neruda, Estr: Concierto "LS and His Students", Hadas Gur (mezzosoprano) y ensamble, Haim Mahlev (director), Rebeca Crown Hall, Jerusalén, 18 de octubre de 2004, Ed: IMI 7548 (16 de julio de 2004), Obs: "for E.B., "Tel Aviv 17-VII, 29-VII,31-VII, 3-VIII-2003", Israel.

[O-314] Intermezo, 2004, cto mand, Dur: 7', Ed: 7547 (16 de junio de 2004). Obs: "To Yuval and Noam on their birthday" (A Yuval y Noam en sus cumpleaños), "10-II-2004", Israel.

[O-315] Duetto, 2004, 2 arp, Dur: 7’, Ed: IMI 7546 (16 de junio de 2004), Obs: “15-II-2004”, Israel.

[O-316] Sunrise (Amanecer), 2004, orq: picc, 2 fl, 2 ob, ci, 2 cl, 1 clb, 2 fg, 2 cfg, 3 tpt, 4 cor, 3 tbn, 1 tu, perc (3 percusionistas), 12 vn I, 12 vn II, 10 va, 8 vc, 6 cb, Dur: 11', Ed: IMI 7571 (22 de noviembre de 2004), Obs: "And the sun shall rise again" (Y el sol se levantará de nuevo), "28-II-2004", Israel.

[O-317] Sunset (Ocaso), 2004, orq: 4 fl, 4 ob, 4 cl, 4 fg, 4 cor, 4 tpt, 3 tbn, 1 tu, perc (4 percusionistas), 14 vn I, 14 vn II, 10 va, 10 vc, 8 cb, Dur: 7', Ed: IMI 7572 (22 de noviembre de 2004), Obs: “And the sun fall down again" (Y el sol se pone de nuevo), "Tel Aviv 30-VI-2004", Israel.

[O-318] Septet, 2004, fl, ob, cl, fg, vn, va, vc, Dur: 11', Ed: IMI 7614 (6 de abril de 2005), Obs: Israel. 
[O-319] Introitus, 2004, gran orq: 3 fl, 3 ob, 3 cl, 3 fg, 4 tpt, 4 cor, 4 tbn, perc (5 percusionistas), arp, cel, 12 vn I, 10 vn II, 8 va, 6 vc, 4 cbs, Dur: 7', Ed: IMI 7611 (6 de abril de 2005), Obs: "Tel Aviv 23 VII, 2004", Israel.

[O-320] Three Psalms and a Prayer (Tres salmos y una oración), 2004, comx (4 S, 4 A, 4 T, 4 B), Dur: 7', Text: Biblia (Salmos 57, 2; 61, 2;59,17 (Latín), Ed: IMI 7563 (9 de septiembre de 2004), Obs: "To my son Eli Schidlowsky" (A mi hijo Eli Schidlowsky), "7-7-2004”, Israel.

[O-321] Quartet, 2004, fl, ob, cl, fg, Dur: 7', Ed: IMI 7561 (9 de septiembre de 2004).Obs: “Tel Aviv 4-VIII-2004", Israel.

[O-322] Libera me Domini (Librame oh Dios), 2004, co a cappella (S, A, T, B), Dur: 7', Text: Misa cristiana, Ed: 7569 (22 de noviembre de 2004), Obs: "Tel Aviv 16-8-2004", Israel.

[O-323] Partita, 2004, cl, Dur: 5', Estr: Jonnatan Hadas, Tel Aviv, 2004 Ed: IMI 7570 (22 de noviembre de 2004), Obs: "To the memory of Uri Adelman" (A la memoria de Uri Adelman), "Tel Aviv, 109-2004", Israel.

[O-324] Quintet, 2004, fl, ob, cl, fg, cor, Dur: 7', Ed: IMI 7610 (28 de marzo de 2005), Obs: "Tel Aviv 24-9-2004", Israel.

[O-325] Elegie, 2004, vn I, vn II, va, vc, cb, Dur. 7', Ed: IMI 7576 (9 de enero de 2005), Obs: "In memoriam my son Elias Schidlowsky" (A la memoria de mi hijo Elías Schidlowsky), Israel.

[O-326] Job, 2004, gran orq: picc, 2 fl, 2 ob, ci, 2 cl, clb, 2 fg, 1 cfg, 4 tpt, 4 cor, 3 tbn, 1 tu, perc (5 percusionistas), 12-14 vn I, 12-14 vn II, 8-10 va, 8-10 vc, 4-8 cb, Dur: 14', Ed: IMI 7618 (8 de junio de 2005), Obs: "For my daughter Judith Schidlowsky" (Para mi hija Judith Schidlowsky), “Tel Aviv 31-XII-2004", Israel.

[O-327] Sextett, 2004, ob, fg, vc, perc (3 percusionistas), Dur: 7', Ed: IMI 7613 (6 de abril de 2005), Obs: "Tel Aviv 4-XII-2004", Israel.

[O-328] L'inferno, 2005, gran orq: picc, 2 fl, 2 ob, 2 cl, clb, 2 fg, cfg, 4 tpt, 4 cor, 4 tbn, tu, perc (4 percusionistas), 12-14 vn I, 12-14 vn II, 8-10 va, 8-10 vc, 6-8 cb, Dur: 11', Ed: IMI 7619 (8 de junio de 2005), Obs: "Tel Aviv 25-I-2005", Israel.

[O-329] Partita, 2005, vc, Dur: 4', Ed: IMI 7612 (6 de abril de 2005), Obs: “Tel Aviv 3-II-2005”, Israel.

[O-330] Resonances (Resonancias), Trío, 2005, vn, vc, pf, Dur: 11', Ed: IMI 7609 (29 de marzo de 2005), Obs: "Tel Aviv 14-II-2005", Israel.

[O-331] Toccata, 2005, pf, perc (1 percusionista), Dur: 7-14', Ed: IMI 7631 (22 de diciembre de 2005), Estr: Concierto en Homenaje al compositor en sus 75 años, Martin Schneuing (piano), Hjórleifur Jónsson (percusión), Berlín, 16 de septiembre de 2006, Fon: CD ma34, LC 00754, musikart ingo schulz (www.musik-art.de), grabación del estreno, Obs: Israel.

[O-332] Three Pieces (Tres piezas), 2005, co a cappella, Dur: 10', Text: Paul Celan, Ed: IMI 7633 (22 de diciembre de 2005), Obs: "Tel Aviv 27-III-2005", Israel.

[O-333] Two Songs (Dos canciones), 2005, S, cl, fg, pf, vn, vc, Dur: 7', Text: Dylan Thomas, Estr: Ensemble Meitar, Gil Raveh (director), Tel Aviv, 23 de octubre de 2006, Ed: IMI 7634 (22 de diciembre de 2005), Obs: Israel.

[O-334] Nocturno, 2006, narr, orq: 2 fl, 2 ob, ci, 2 cl, clb, 2 fg, cfg, 4 tpt, 4 cor, 3 tbn, tu, perc (4 percusionistas), arp, cel, 12 vn I, 12 vn II, 10 va, 8 vc, 6 cb, Dur: 10', Text: Pablo Neruda (Canto General), Ed: IMI 7720 (18 de mayo de 2006), Obs: "Tel Aviv 16-3-2006”, Israel.

[O-335] In memoriam György Ligeti, 2006, orq: 4 fl, 4 ob, 4 cl, 4 fg, 4 tpt, 4 cor, 3 tbn, tu, arp, cel, 14 vn I, 14 vn II, 10 va, 10 vc, 8 cb, Dur: 10', Ed: IMI 7857 (10 de diciembre de 2008), Obs: "Tel Aviv 28-6-2006”, Israel.

[O-336] Two Songs (Dos canciones), 2006, V, cl, vn, vc, Dur: 7', Text: William Blake y Walter SawageLandor, Estr: Uta Buchheister (mezzosoprano), Matthias Badzcong (clarinete), Eva Polster 
(violín), Regine Zimmermann (violonchelo), Berlín, 23 de junio de 2007, Ed: IMI 7719 (18 de mayo de 2006), Israel.

[O-337] The Voice of the Wind (La voz del viento), 2006, orq: $3 \mathrm{fl}, 3 \mathrm{ob}, 3 \mathrm{cl}, 3 \mathrm{fg}, \mathrm{cfg}, 3 \mathrm{tpt}, 3 \mathrm{cor}, 3$ tbn, tu, perc (3 percusionistas), 12 vn I, 12 vn II, 10 va, 8 vc, 6 cb, Dur: 10', Ed: IMI 7770 (28 de febrero de 2007), Obs: "Tel Aviv 14-VI-2006", Israel.

[O-338] Constellations (Constelaciones), 2006, orq: 4 fl, 4 ob, 4 cl, 4 fg, 4 tpt, 4 cor, 3 tbn, tu, perc (4 percusionistas), arp, cel, 14 vn I, 14 vn II, 10 va, 10 vc, 8 cb, Dur: 7', Ed: IMI 7771 (28 de febrero de 2007), Obs: "Tel Aviv 27-X-2006", Israel.

[O-339] Eros $\mathcal{E}$ Thanatos, 2007, orq: picc, 2 fl, 2 ob, ci, 2 cl, clb, 2 fg, cfg, 4 tpt, 4 cor, 3 tbn, tu, perc (3 percusionistas), arp, cel-pf, 12-14 vn I, 12-14 vn II, 10-12 va, 8-10 vc, 6-8 cb, Dur: 10', Ed: IMI 7838 (5 de mayo de 2008), Obs: "28-II-2007", Israel.

[O-340] Horror Vacui (Horror al vacío), 2007, orq ca: narr, fl (fl en Sol, picc), ob (ci), cl (cl picc, clb), perc (1 percusionista), Dur: 10', Estr: Benjamin Boresch (recitante), Franciska Leonhardt (flauta, flauta en Sol, piccolo), Thamar Müller (oboe, corno inglés), Martin Ackermann (clarinete, clarinete piccolo, clarinete bajo), Simon Lessing (percusión,) Elias Corrinth (director), Leipzig 20 de abril de 2011, Ed: IMI 7836 (5 de mayo de 2008), Obs: "7-06-2007", Israel.

[O-341] The March of the Ants (La marcha de las hormigas), 2007, 2 perc, Dur: libre, Ed: IMI 7834 (5 de mayo de 2008), Obs: "To Judith and David" (A Judith y David), "Tel Aviv 9-9-2007", Israel.

[O-342] Sul do (Sobre el Do), 2007, orq: fl picc, fl en Sol, cl picc, clb en sib, tpt en Do, cor, tu, perc (5 percusionistas), 7 vn I, 7 vn II, 5 va, 5 vc, 4 cb, Dur: 7’, Ed: IMI 7837 (5 de mayo de 2008), Obs: "In memoriam Marcel Marceau", "Tel Aviv 28-9-2007”, Israel.

[O-343] The Seventh Eye (El Séptimo Ojo), 2008, orq, 4 fl, 4 ob, 4 cl, 4 fg, 4 tpt, 4 cor, 3 tbn, tu, perc (4 percusionistas), arp, cel, 14 vn I, 14 vn II, 10 va, 10 vc, 8 cb, Dur: 7', Ed: IMI 7839 (5 de mayo de 2008), Obs: "In memoriam Maurice Bejart”, “Tel Aviv 1-I-2008”, Israel.

[O-344] Three Songs (Tres canciones), 2008, S, fl, cl, fg, vn, vc, pf, Dur: 7', Text: Giuseppe Ungaretti (Cantatto senza parole) (cantado sin palabras), Estr: Claimont Auditorium, Meitar Ensamble, Karin Ben-Yosef (director), Jaffo, Israel, 27 de diciembre de 2008, Ed: IMI 7835 (5 de mayo de 2008), Obs: "Dedicated to Meitar Ensamble" (Dedicado al Ensamble Meitar), "Tel Aviv 14-022008”, Israel.

[O-345] Monolog, 2008, gui, narr, Dur: 5', Text: Nicolás Guillén (Iba yo por un camino), Estr: Rubén Seroussi (guitarra y narrador), Auditorio Teiva, Jaffo, Israel, 20 de febrero de 2010. Rubén Serousi, (guitarra y narrador), Auditorio Nacional del Sodre Dra. Adela Reta, Montevideo, Uruguay, 16 de setiembre de 2010, Ed: IMI 7854 (10 de diciembre de 2008), Obs: Israel.

[O-346] Diagram, 2008, mand, gui, Dur: 7', Estr: Alon Sariel (mandolina), Uri Jacob (guitarra), Plucked Instruments and more., Israeli-Music-Festival, Auditorium, Performing Arts Centre, Rishon LeZion, Israel, 21 de septiembre de 2015, Ed: IMI 7853 (10 de diciembre de 2008), Obs: "28-06-2008", Israel.

[O-347] Actions 77, 2008, orq: 4 fl, 4 ob, 4 cl, 4 fg, 4 tpt, 4 cor, 3 tbn, tu, perc (4 percusionistas), arp, cel, 12-14 vn I, 12-14 vn II, 8-10 va, 8-10 vc, 6-8 cb, Dur: 7', Ed: IMI 7856 (10 de diciembre de 2008), Obs: "21-07-2008", Israel.

[O-348] Prelude, 2008, mand, Dur: 7', Ed: IMI 7852 (10/12/2008), Obs: “21-06-2008”, Israel.

[O-349] Two Psalms (Dos salmos), 2008, comx a cappella, Dur: 7', Text: Biblia, Salmos 23,1-6; 144,3-4, Ed: IMI 7855 (10 de diciembre de 2008), Obs: “Tel Aviv 23-09-2008”, Israel.

[O-350] Love Song (Canción de amor), 2008, S, orq: 2 fl, 2 ob, 2 cl, 2 fg, 1 cfg, 2 tpt, 2 cor, 2 tbn, tu, perc (3 percusionistas), arp, pf-cel, 14 vn I, 12 vn II, 10 va, 8 vc, 6 cb, Dur: 7', Text: compositor, Ed: IMI 7861 (23 de diciembre de 2008), Obs: Tel Aviv "21-XI-2008”, Israel.

[O-351] Ritual Dance (Danza ritual), 2009, orq: picc, 2 fl, 2 ob, 2 cl, clb, 2 fg, cfg, 2 tpt, 2 cor, 3 tbn, tu, perc (4 percusionistas), vn I, vn II, va, vc, 8 cb, Dur: 7', Ed: IMI 7889 (7 de diciembre de 2009), Obs: "Tel Aviv 16-III-2009", Israel. 
[O-352] Soledad, 2009, V, ob, cor, vc, Dur: 5', Text: quechua, Ed: IMI 7888 (7 de diciembre de 2009), Obs: "28-III-2009", Israel.

[O-353] Portray (Retratar), 2009, orq: 2 fl, 2 ob, 2 cl, 2 fg, cfg, 2 tpt, 2 cor, 2 tbn, tu, perc (3 percusionistas), arp, pf-cel, vn I, vn II, va, vc, cb, Dur: 7-10', Ed: IMI 7883 (2 de diciembre de 2009), Obs: "25-08-2009", Israel.

[O-354] Lautaro, 2009, narr, orq: 2 fl, 2 ob, 2 cl, 2 fg, 2 tpt, 2 cor, 2 tbn, perc (3 percusionistas), 7 vn I, 7 vn II, 6 va, 5 vc, 3 cb, Dur: 10', Text: Pablo Neruda (Canto General: Los libertadores), Estr. Centro Cultural Gabriela Mistral, Pablo Oyanedel (narrador), Orquesta de Cámara de Chile, Juan Pablo Izquierdo (director), Santiago de Chile, 5 de septiembre de 2010. Teatro Universidad de Chile, Ernesto Ottone R. (narrador), OSCH, Juan Pablo Izquierdo (director), Santiago de Chile, 6-7 de septiembre de 2013, Ed: IMI 7917 (5 de abril de 2010). Obs: "Dedicated to Bernardita de los Ángeles" (Dedicado a Bernardita de los Ángeles), "Tel Aviv 24-11-2009”, Israel.

[O-355] Mahleriana, 2011, orq: 2 fl, 2 ob, 2 cl, 2 fg, cfg, 2 tpt, 2 cor, 2 tbn, tu, perc (3 percusionistas), arp, pf-cel, vn I, vn II, va, vc, cb, Dur: 10', Estr: Orquesta Sinfónica Universidad de Concepción, Doron Salomon (director), Concepción, Chile, 16 de junio de 2012, Ed: IMI 7951 (10 de agosto de 2011), Obs: "In memoriam Gustav Mahler on the 100 years anniversary of his death" (In memoriam Gustav Mahler en el centenario de su muerte), "Tel Aviv 26-II-2011”, Israel.

[O-356] Solo, 2011, co ca o 4 sol, Dur: 7', Text: Hilel (hebreo), Ed: IMI 7977 (7 de mayo de 2012), Obs: “Tel Aviv 11-07-2011”, Israel.

[O-357] Capricho, 2011, orq: 2 fl, 2 ob, 2 cl, 2 fg, cfg, 4 tpt, 4 cor, 2 tbn, tu, perc (3 percusionistas), arp, pf-cel, vn I, vn II, va, vc, cb, Dur: 14', Ed: IMI 7926 (30 de julio de 2012), Obs: "Tel Aviv 24XII-2011", Israel.

[O-358] Twilight (Crepúsculo), 2011, ob, cl, cor, vc, Dur: 8', Ed: IMI 7978 (7 de mayo de 2012), Obs: "Tel Aviv 14-I-2012", Israel.

[O-359] Intermezzo, 2012, fl, ob, 2 vn, va, vc, cb, pf, Dur: 10', Ed: IMI 7980 (7 de mayo de 2012), Obs: “Al Centro de Estudios de la Composición 'Matta 365'”, Viña del Mar, Chile, “Tel Aviv 7-02-2012”, Israel.

[O-360] Sexteto, 2012, fl, ci, clb, arp, vc, cb, Dur: 7', Ed: IMI 7979 (7 de mayo de 2012), Obs: Motto: Salmo 23,4, "Tel Aviv 19-II-2012”, Israel.

[O-361] Obelisk, 2012, orq: 4 fl, 4 ob, 4 cl, 4 fg, 4 tpt, 4 cor, 3 tbn, tu, perc (4 percusionistas), arp, pf-cel, 14 vn I, 14 vn II, 10 va, 10 vc, 8 cb, Dur: 10', Ed: IMI 7997 (30 de julio de 2012), Obs: "In memoriam those who paid with their lives for Peace and Humanity" (A la memoria de aquellos que ofrendaron su vida por la paz y la humanidad), "Tel Aviv 4-03-2012", Israel.

[O-362] Shakesperiana, 2012, 2 vn, 2 VV, Dur: 10', Text: William Shakespeare (fragmentos de diferentes obras), Ed: IMI 8001 (30 de julio de 2012), Obs: "Dedicated to Yair and Egal Kless" (Dedicada a Yair y Egal Kless), "21-04-2012”, Israel.

[O-363] Y álzase el hombre, 2012, narr, perc, Dur: 10', Text: “César Vallejo: Resurrection”, Ed: IMI 8000 (30 de julio de 2012), Obs: "3-05-2012", Israel.

[O-364] Farewell (Despedida), 2012, fl, Dur: 5', Estr: Noam Lion, Nueva York, 2012, Ed: IMI 7999 (30 de julio de 2012), Obs: "Dedicated to Noam Lion" (Dedicado a Noam Lion), "21-V-2012", Israel.

[O-365] Izkor, 2012, vn, Dur: libre, Estr: Lateinamerikanische Nacht (Noche latinoamericana), Hochschule für Musik und Darstellende Musik, Johann Wolfgang Goethe-Universität Frankfurt am Main, Gustavo Vergara (violín), Frankfurt am Main 19 de octubre de 2014. Temporada UTEM 2015, Gustavo Vergara (violín), Santiago de Chile, 17 de agosto de 2015. Ed: IMI 7998 (30 de julio de 2012), Obs: "In memoriam my father and mother" (A la memoria de mi padre y mi madre), Israel.

[O-366] Exodus, 2012, 4 sol o co a cappella, Dur: 14', Text: Biblia (Éxodo 19,6), Ed: IMI 8003 (17 de enero de 2013), Obs: "Tel Aviv 25-09-2012", Israel. 
[O-367] Desierto 2012, narr, orq: 2 fl, 2 ob, 2 cl, 2 fg, cfg, 2 tpt, 4 cor, 2 tbn, tu, perc (3 percusionistas), arp, pf-cel, 12 vn I, 12 vn II, 10 va, 8 vc, 6 cb, Dur: 7', Text: Pablo Neruda (Canto General, Los libertadores), Ed: IMI. 8004 (7 de octubre de 2013), Obs: "Tel Aviv 28-X-2012”, Israel.

[O-368] Duetto (Vivace, Adagio, Allegro, Andante, Agitato), 2012, 2 fl, Dur: 10', Ed: IMI 8027 (7 de octubre de 2013), Obs: "Tel Aviv 17-12-2012", Israel.

[O-369] Permutaciones, 2012, V o VV con perc (claves, maracas, crótalos, guiro y flexatono), Dur: abierta, Obs: Partitura gráfica, Israel.

[O-370] Hopeless Day (Día de desesperanza), 2013, narr, orq: 4 fl, 4 ob, 4 cl, fg, 4 tpt, 4 cor, 3 tbn, tu, perc (3 percusionistas), arp, pf-cel, 14 vn I, 14 vn II, 10 va, 10 vc, 8 cb, Dur: 14', Text: Biblia, Isaías 24, 17-23, Obs: "Tel Aviv 28-9-2012", Israel.

[O-371] Musical Landscapes (Paisajes musicales), 2013, orq: 2 fl, $2 \mathrm{ob}, 2 \mathrm{cl}, 2 \mathrm{fg}, \mathrm{cfg}, 2 \mathrm{tpt}, 4 \mathrm{cor}, 2 \mathrm{tbn}$, tu, perc (3 percusionistas), arp, pf-cel, 12 vn I, 12 vn II, 10 va, 8 vc, 8 cb, Dur: 7-10', Estr: Temporada 2015, Concierto $N^{\circ}$ 5, Teatro Municipal de Santiago, Orquesta Filarmónica de Santiago, Paolo Bortolameolli (director), Santiago de Chile, 5 y 6 de junio de 2015, Ed: IMI 8032 (7 de octubre de 2013), Obs: "Tel Aviv 4-II-2013”, Israel.

[O-372] The Day of Rage (El día de la ira), 2013, orq: $2 \mathrm{fl}, 2 \mathrm{ob}, 2 \mathrm{cl}, 2 \mathrm{fg}, \mathrm{cfg}, 2 \mathrm{tpt}, 2 \mathrm{cor}, 2 \mathrm{tbn}$, tu, perc (3 percusionistas), arp, pf-cel, 12 vn I, 12 vn II, 10 va, 8 vc, 6 cb, Dur: 7’, Ed: IMI 8034 (30 de diciembre de 2013), Obs: "A legend” (Una leyenda), “Tel Aviv 17-8-2013”, Israel.

[O-373] Memorial of Yesterday's Dreams (Memorial de los sueños de ayer), 2013, orq: 2 fl, 2 ob, 2 cl, 2 fg, cfg, 2 tpt, 2 cor, 2 tbn, tu, perc (3 percusionistas), arp, pf-cel, 12 vn I, 12 vn II, 10 va, 8 vc, 6 cb, Dur: 7', Obs: Motto: Cantar de los Cantares, 7,6, "Tel Aviv 2-9-2013", Israel.

[O-374] Presage (Presagio), 2013, orq: 2 fl, 2 ob, 2 cl, 2 fg, cfg, 2 tpt, 2 cor, 2 tbn, tu, perc (3 percusionistas), arp, pf-cel, vn I, vn II, va, vc, cb, Dur: 7', Ed: IMI 8151 (6 de diciembre de 2015), Obs: "28-11-2013", Israel.

[O-375] Epigram, 2013, orq: 2 fl, 2 ob, 2 cl, 2 fg, cfg, 2 tpt, 2 cor, 2 tbn, tu, perc (3 percusionistas), arp, pf-cel, vn I, vn II, va, vc, cb, Dur: 7', Ed: IMI 8150 (6 de diciembre de 2015), Obs: “14-12-2013”, Israel.

[O-376] The Valley of Dry Bones (El valle de los huesos secos), 2014, orq: 2 fl, 2 ob, 2 cl, 2 fg, cfg, 2 tpt, 2 cor, 2 tbn, tu, perc (3 percusionistas), arp, pf-cel, 12 vn I, 12 vn II, 10 va, 8 vc, 6 cb, Dur: 7', Ed: IMI 8149 (6 de diciembre de 2015), Obs: Motto: Exequiel 37,1-4, "Dedicated to my daughter Judith StahlSchidlowsky” (Dedicada a mi hija Judith Stahl-Schidlowsky), Tel Aviv, 25 de enero de 2014, Israel.

[O-377] Journey to the End of the Night (Viaje al fin de la noche), 2014, orq: $2 \mathrm{fl}, 2 \mathrm{ob}, 2 \mathrm{cl}, 2 \mathrm{fg}, \mathrm{cfg}, 2 \mathrm{tpt}$, 2 cor, 2 tbn, tu, perc (4 percusionistas), arp, pf-cel, 12 vn I, 12 vn II, 10 va, 8 vc, 6 cb, Dur: 14', Ed: IMI 8148 (6 de diciembre de 2015), Obs: "Tel Aviv 29-X-2014", Israel.

[O-378] In memoriam Fernando Rosas, 2014, orq: 2 fl, 2 ob, 2 cl, 2 fg, 2 tpt, 2 cor, perc (1 percusionista), 6 vn I, 5 vn II, 4 va, 3 vc, 2 cb, Dur: 7', Ed: IMI 8096 (6 de enero de 2015), Obs: "Tel Aviv 15-9-2014", Israel.

[O-379] Valparaíso, 2014, narr, orq: 2 fl, $2 \mathrm{ob}, 2 \mathrm{cl}, 2$ fg, cfg, 2 tpt, 2 cor, 2 tbn, tu, perc (4 percusionistas), arp, pf-cel, 12 vn I, 12 vn II, 10 va, 8 vc, 6 cb, Dur: 14', Text: Álvaro Gallegos, Ed: IMI 8097 (6 de enero de 2015), Obs: "Dedicado a Álvaro Gallegos", "Tel Aviv 20-X-2014”, Israel.

[O-380] Luces y sombras, 2015, orq: 2 fl, 2 ob, 2 cl, 2 fg, cfg, 2 tpt, 2 cor, 2 tbn, tu, perc (4 percusionistas), arp, pf-cel, 12 vn I, 12 vn II, 10 va, 8 vc, 6 cb, Dur: 8', Ed: IMI 8147 (6 de diciembre de 2015), Obs: "To my son David” (A mi hijo David), "16-2-15”, Israel.

[O-381] The World of the See (El mundo del mar), 2015, orq: $2 \mathrm{fl}, 2 \mathrm{ob}, 2 \mathrm{cl}, 2 \mathrm{fg}, \mathrm{cfg}, 2 \mathrm{tpt}, 2$ cor, 2 tbn, tu, perc (4 percusionistas), arp, pf-cel, 12 vn I, 12 vn II, 10 va, 8 vc, 6 cb, Dur: 8-9', Ed: IMI 8146 (6 de dciembre de 2015), Obs: Moto: fragmento de "Fern Hill" de Dylan Thomas, "Tel Aviv 9/10.4.2015", Israel. 
[O-382] Memoria, 2015, orq: 2 fl, 2 ob, 2 cl, 2 fg, cfg, 2 tpt, 2 cor, 2 tbn, tu, perc (4 percusionistas), arp, pf-cel, 12 vn I, 12 vn II, 10 va, 8 vc, 6 cb, Dur: 14', Ed: IMI 8152 (6 de diciembre de 2015), Obs: "Until I die he will not leave my side" (Hasta que yo muera él no dejará mi lado) (Elegy, Dylan Thomas), "In memory of my son Elías Schidlowsky" (A la memoria de mi hijo Elías Schidlowsky), “Tel Aviv, 21-4-2015”, Israel.

[O-383] Chamber Concert (for 14 players) (Concierto de cámara para 14 intérpretes), 2015, fl, ob, cl, fg, tpt, cor, tbn, perc (2 percusionistas), 1 vn I, 1 vn II, 1 va, 1 vc, 1 cb, Dur: 7'-14', Obs: “21-VII-2015”, Israel.

[O-384] Obelisk II, para gran orquesta, 2015, 4 fl, 4 ob, 4 cl, 4 fg, 4 tpt, 4 cor, 3 tbn 1 tu, arp, cel, 4 timp (1 ejecutante), perc (3 ejecutantes), 14 vn I, 14 vn II, 10 va, 10 vc, 8 cb, Dur: 7 minutos, Obs: "In memoriam of the victims of terrorism" (En memoria de las víctimas del terrorismo), "Tel Aviv, 25-IX-2015”.

[O-385] Partita - esquema para orquesta, 2015, 2 fl, 2 ob 2 cl, 2 fg, 1 cfg, 2 tpt, 2 cor, 2 tbn, 1 tu, 4 timp, perc (4 ejecutantes), arp, cel/pf, 12 vn I, 12 vn II, 10 va, 8 vc, 6 cb, Dur: 7 minutos, Obs: "Música es un atentado al sonido" (palabras de León Schidlowsky al recibir el Premio Nacional de Música 2014), "Tel Aviv, VII-X-2015”.

[O-386] Gedanken - Thoughts (Pensamientos), 2015, S, A, T, B (o coro), Dur: 7', Text: Paul Celan, Obs: "Tel Aviv, 28-X-2015”, Israel.

[O-387] Poem, 2015, 2 fl, 2 ob, 2 cl, 2 fg, 2 tpt, 2 cor, 2 tbn, 4 timp, perc (3 percusionistas) , 7-14 vn I, 7-14 vn II, 5-10 va, 5-10 vc, 4-8 cb, Dur: 8-10 minutos, Obs: "A mi madre”, “Tel Aviv, 22-XI-2015”, Israel. 